TITLE:

\title{
Localized Wrinkle Behavior near Fixed Boundaries in Flat and Cylindrical Membranes
}

$\operatorname{AUTHOR}(S)$ :

Senda, Kei; Petrovic, Mario; Nakanishi, Kei

\section{CITATION:}

Senda, Kei ... [et al]. Localized Wrinkle Behavior near Fixed Boundaries in Flat and Cylindrical Membranes. Journal of Spacecraft and Rockets 2015, 52(4): 1074-1090

\section{ISSUE DATE:}

2015-07

URL:

http://hdl.handle.net/2433/202089

\section{RIGHT:}

This is the author's version of the work. The definitive version is available online at: http://arc.aiaa.org/doi/10.2514/1.A33018.; This is not the published version. Please cite only the published version.; この論文は出版社版でありません。引用の際には出版社版 をご確認ご利用ください。 


\title{
Localized wrinkle behavior near fixed boundaries
}

\section{in flat and cylindrical membranes}

\author{
Kei Senda*, Mario Petrovic ${ }^{\dagger}$, and Kei Nakanishi ${ }^{\ddagger}$ \\ Kyoto University, Kyoto 606-8501, Japan
}

This study analyzes wrinkle behavior in both flat and cylindrical membranes undergoing shear displacement. The goal is to establish a relationship between the wrinkle behavior in the two types of membranes and understand how different geometric properties affect the behavior. The analysis is an equilibrium path tracking analysis using the finite element method. By applying cyclic boundary conditions to a flat membrane, a degree of similarity in the wrinkle behavior can be established between flat and cylindrical membranes. In both membranes, the initial wrinkles cover the entire membrane. These wrinkles are followed by smaller, localized wrinkles, referred to as collapsed sections. These collapsed sections form on existing wrinkles. The generation of new wrinkles, by splitting the existing wrinkles, is controlled by collapsed sections and is the same in both types of membranes. For the cylindrical membrane, the curved shape results in a more controlled wrinkle behavior. This is represented by fewer bifurcation points that require larger values of shear to occur. Secondly, the collapsed section frequency and position is fixed with respect to the wrinkle frequency and position. The cause of simplified and controlled wrinkle behavior is analyzed, and the control mechanism is explained.

\footnotetext{
* Professor, Department of Aeronautics and Astronautics, Graduate School of Engineering, senda@kuaero.kyotou.ac.jp, Senior Member AIAA.

† Graduate Student, Department of Aeronautics and Astronautics, Graduate School of Engineering, mapetrov@live.com, Member AIAA

¥ Graduate Student, Department of Aeronautics and Astronautics, Graduate School of Engineering, keinakanishi5@gmail.com, Member AIAA
} 


\section{Nomenclature}

$a$ = length, $\mathrm{m}$

$b=$ width, $\mathrm{m}$

$D=$ Flexural rigidity, $\mathrm{Nm}^{2}$

$d=$ shell thickness, $\mathrm{m}$

$E=$ Young's Modulus, GPa

$f=$ force, $\mathrm{N}$

$M=$ shell section moment, $\mathrm{Nm} / \mathrm{m}$

$N=$ shell section force, $\mathrm{N} / \mathrm{m}$

$q=$ displacement, $\mathrm{m}$

$R=$ radius, $\mathrm{m}$

$t=$ time, $\mathrm{s}$

$u=$ displacement, $\mathrm{m}$

$\delta f=$ imposed force, $\mathrm{N}$

$\delta u=$ imposed displacement, $\mathrm{m}$

$\epsilon=$ membrane strain

$\kappa=$ curvature

$\lambda=$ eigenvalue

$\nu=$ Poisson's ratio

$\phi=$ eigenvector

$\sigma=$ membrane stress, $\mathrm{N} / \mathrm{m}^{2}$

\section{Introduction}

Inflatable structures are often considered for the design of larger space vehicles because such structures satisfy weight and storage requirements[1]. The inflatable structures considered in the study are deployable membrane structures. Initially, the membrane is folded. By applying loads to the membrane, it is unfolded or inflated, deployed into its desired shape, and rigidified.

The research goal of this study is the stable deployment of a structure into a prescribed shape 
along a planned trajectory. However, because the structure is a membrane, with many degrees of freedom, the prediction of membrane behavior is difficult. The term membrane behavior, refer to the displacement of membrane points under the effect of external loads. A deployment experiment using an inflatable tube[2], investigated an origami-like pattern to facilitate predictable deployment. In the experiment, the generation of wrinkles was observed due to local buckling. Buckling occurs after a bifurcation[3-6] that may result in an unstable structure. The resulting undetermined states make predicting stable deployment difficult. Therefore, understanding membrane wrinkling and its bifurcations is essential.

In this study, a wrinkle is a finite deformation of the membrane that occurs in a direction different from the direction of the load applied to the structure. Generally, this results in compression of the membrane which leads to buckling.

An equilibrium state of the membrane, represented by an equilibrium point, is defined as a state of a membrane for a static load or an imposed displacement. Here, the static load or the imposed displacement can be considered as path parameters. By changing the value of the path parameter, different equilibrium points are obtained. Connecting multiple, successive equilibrium points results in a formation of an equilibrium path. When a bifurcation occurs, the membrane undergoes a qualitative change of the equilibrium state. This qualitative change is usually represented by the generation of wrinkles. Additionally, a bifurcation may result in path branching, i.e. multiple paths exist after bifurcation. Therefore, in order to deploy a membrane structure along a planned trajectory, an understanding of the bifurcation structure and the equilibrium paths is necessary.

Membrane wrinkling studies have focused mostly on certain effects on characteristics, i.e. gravity[8], creases[9], stress concentrations[10], etc. In general, wrinkling is analyzed as a membrane behavior event, and wrinkled geometry is computed for specific loads. The goal of this study is to obtain a comprehensive understanding of membrane wrinkling. For that purpose, several wrinkle generations, multiple wrinkle configurations, and wrinkle interactions are considered.

In previous studies[4, 11], a flat rectangular membrane with free boundary conditions was sheared. In Wong and Pellegrino[5] and Cerda[7], the topic is approached from a more theoretical standpoint used to predict wrinkle amplitude and wavelength. However, the mechanism behind 
wrinkling is not discussed in detail. Finally, in Wong and Pellegrino[6], a wide range of numerical results were shown. In addition, Senda, et al. [12] showed wrinkle behavior for relatively small values of imposed shear. The study focused on the the generation of wrinkles without bifurcations. It was shown that at smaller values of shear, parts of the membrane remain flat while other parts of the membrane have wrinkles. Those existing wrinkles then generate new wrinkles on the flat part without bifurcations.

However, once the membrane becomes fully wrinkled, localized wrinkles start to form along the fixed boundaries of the membrane. These localized wrinkles form on existing wrinkles, grow with increasing shear, and split the original wrinkle. However, the use of free boundaries results in an uneven distribution and shape of wrinkles. This made the observation of wrinkle generation difficult.

In this study, to eliminate the uneven shape and distribution of wrinkles, a flat rectangular membrane is modeled using cyclic boundary conditions. However, this type of membrane is not practical. Therefore, a cylindrical membrane is modeled using the same geometry as the flat membrane. By making the shear displacement sufficiently large, both membranes are fully wrinkled. Further increase in shear displacement results in small localized wrinkles generated near the fixed boundaries on existing wrinkles. These small wrinkles will be referred to as collapsed sections to distinguish them from the larger wrinkles. By increasing the shear displacement further, the collapsed sections expand along the length of the wrinkles. At a critical size, the sections undergo an unstable expansion. They join in the middle of the existing wrinkle, split it, and result in two new wrinkles. This behavior repeats with further increases in shear displacement and represents the basic behavior for increase in wrinkle number when the membrane is fully wrinkled.

By analyzing both the flat membrane and the cylindrical membrane, it is possible to establish similarities and differences in behavior. Using displacement and stress fields in the membrane, the mechanism behind the formation of localized wrinkles is shown. The comparison of the two membranes also shows that the cylindrical membrane has a natural mechanism for controlling local wrinkle behavior. This mechanism is explained, and its potential application in flat membranes is discussed. 
The rest of this paper is organized as follows. Section II sets up the FEM model of the rectangular membrane using ABAQUS, a FEM software package, the path analysis method and the asymptotic theory to a load parameter. Section III shows the analysis method. Section IV discusses membrane and solution symmetry. Section V presents some numerical simulations and discusses the results. Section VI offers some concluding remarks.

\section{Modeling and problem}

\section{A. Analyzed object and model}

This study analyzes two types of membranes; a flat membrane with cyclic boundary conditions and a cylindrical membrane. To maximize the degree of similarity between the two types of membranes, similar geometry is used. The material is Kapton and its properties are listed in Table 1. The choice of a Kapton membrane comes from the flat membrane wrinkling data, e.g. Wong and Pellegrino[4] and Inoue[11].

The flat membrane analyzed in this study is illustrated in Fig. 1. The ratio of the horizontal to the vertical lengths, $a: b$, is $3: 1$. Fixed boundaries are set for the top and bottom edges, and cyclic boundaries for the left and right edges. The fixed top edge is then subjected to a prescribed displacement. Gravity and imperfections are not considered.

The origin of the $(x, y, z)$ coordinate system is fixed at the membrane center of mass in the undeformed state, i.e. no external loads are applied. Coordinates $(x, y, z)$ refer to a position on the membrane prior to deformation, and they do not vary with deformation. Section AA' is defined at

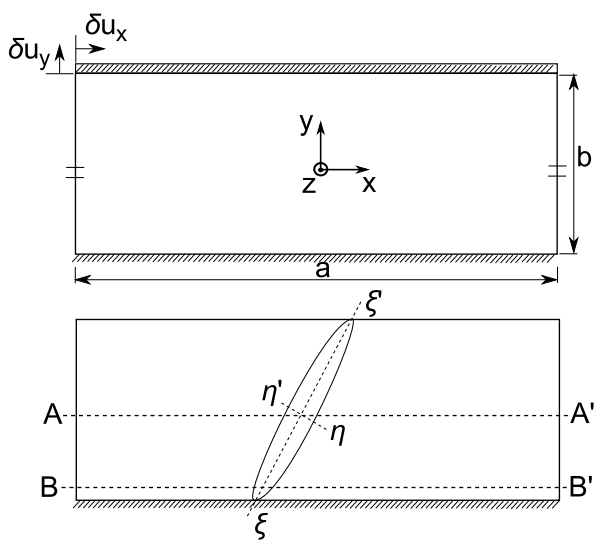

Fig. 1 Sheared flat membrane.

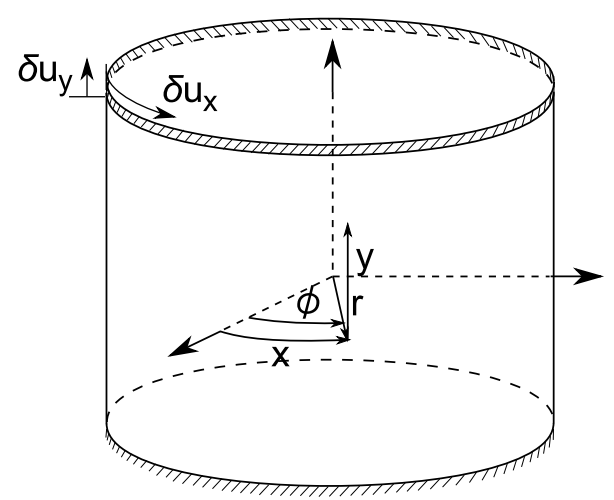

Fig. 2 Sheared cylindrical membrane. 
Table 1 Membrane material properties.

\begin{tabular}{ccc}
\hline \hline Membrane width & $a$ & $0.30[\mathrm{~m}]$ \\
Membrane height & $b$ & $0.10[\mathrm{~m}]$ \\
Membrane thickness & $d$ & $12.5 \times 10^{-6}[\mathrm{~m}]$ \\
Young's Modulus & $E$ & $3.0[\mathrm{GPa}]$ \\
Poisson's ratio & $\nu$ & 0.3 \\
\hline \hline
\end{tabular}

$y=0[\mathrm{~m}]$ and $\mathrm{BB}^{\prime}$ at $y=-0.045[\mathrm{~m}]$.

First, at the top fixed edge, a displacement of $\delta u_{y}=30 \times 10^{-6}[\mathrm{~m}]$ is applied in the $y$-direction and is constant throughout the analysis. This is to maintain consistency with experimental results in Inoue[11]. Then, an imposed displacement $\delta u_{x}$ in the $x$ direction is applied. In this study, $\delta u_{x}$ is increased from $0[\mathrm{~m}]$ to $510 \times 10^{-6}[\mathrm{~m}]$.

Membrane deformation is described by displacements at all points on the membrane. Point displacements are represented by $\left(u_{x}, u_{y}, u_{z}\right)$ in $(x, y, z)$ coordinates. However, the $z$-displacement of points on the mid-plane is of primary interest, and the displacement of a point in $z$-coordinate $u_{z}(x, y, 0)$ is simply expressed by $u_{z}(x, y)$.

Stress and strain are given for the mid-plane. The tensile direction is positive for both stress and strain. Considering plane stress, the $\xi \xi^{\prime}$-axis corresponds to the first principal stress direction and the $\eta \eta^{\prime}$-axis corresponds to the second principal stress direction. When wrinkles exist, the $\xi \xi^{\prime}$-axis in a the wrinkle longitudinal direction is perpendicular to the $\eta \eta^{\prime}$-axis. Both $\xi \xi^{\prime}$ and $\eta \eta^{\prime}$ rotate with the wrinkles and remain perpendicular to each other. A wrinkle is defined as a sinusoidal shaped out-of-plane deformation that occurs between two points where curvature in the $\eta$-direction changes signs.

The geometry of the cylindrical membrane is illustrated by Fig. 2. The length, $a$, of the flat membrane is the circumference of the cylindrical membrane, while the height $b$ applies to both membranes. The ratio $a: b$ is also $3: 1$.

A point in the cylindrical membrane is described by the cylindrical coordinate $(r, \phi, y)$. The points $(r, \phi, y)$ of the cylindrical membrane can also be expressed in terms of $(x, y, z)$ coordinates. By defining $x=R \phi, z=r-R$ where $R=a / 2 \pi,(r, \phi, y)$ coordinates are transformed to $(x, y, z)$ coordinates. The $(x, y, z)$ coordinates of the cylindrical membrane directly correspond to $(x, y, z)$ 
coordinates of the flat membrane. Point displacements in the cylindrical membrane are also the same as the flat membrane in $(x, y, z)$ coordinates. Therefore, the definitions of sections and boundary conditions for the flat membrane also apply to the cylindrical membrane. To avoid confusion, this study uses the $(x, y, z)$ coordinate representation for both membrane models.

The problems dealt with in this study are defined below. Membrane behavior is analyzed by increasing the imposed shear displacement $\delta u_{x}$. The behavior includes wrinkle geometry indicated by displacement $u_{z}(x, y)$, bifurcation structure with respect to increasing shear, and stress. Stress values as well as forces and moments at certain points are used to explain the behavior in more detail. The wrinkle generation mechanism is described by the following analysis.

\section{B. Differential equations}

The first step in the analysis of membrane wrinkling is establishing the governing equations for plates[3]. These equations provide the key properties of the system. For membranes, the KirchhoffLove assumptions for thin plates are adopted. By using the standard plate definitions[3] the plate 
equations are written as:

$$
\begin{aligned}
& \frac{E h}{1-\nu^{2}}\left[\frac{\partial^{2} u_{x}}{\partial x^{2}}+\frac{\partial u_{z}}{\partial x}\left(\frac{\partial^{2} u_{z}}{\partial x^{2}}\right)+\nu\left\{\frac{\partial^{2} u_{y}}{\partial x \partial y}+\frac{\partial u_{z}}{\partial y} \frac{\partial^{2} u_{z}}{\partial x \partial y}\right\}\right] \\
& +\frac{E h}{2(1+\nu)}\left\{\frac{\partial^{2} u_{x}}{\partial y^{2}}+\frac{\partial^{2} u_{y}}{\partial x \partial y}+\frac{\partial^{2} u_{z}}{\partial x \partial y} \frac{\partial u_{z}}{\partial y}+\frac{\partial u_{z}}{\partial x} \frac{\partial^{2} u_{z}}{\partial y^{2}}\right\} \\
& =f_{x}\left(u_{x}, u_{y}, u_{z}\right)=0 \\
& \frac{E h}{1-\nu^{2}}\left[\frac{\partial^{2} u_{x}}{\partial y^{2}}+\frac{\partial u_{z}}{\partial y}\left(\frac{\partial^{2} u_{z}}{\partial y^{2}}\right)+\nu\left\{\frac{\partial^{2} u_{x}}{\partial x \partial y}+\frac{\partial u_{z}}{\partial x} \frac{\partial^{2} u_{z}}{\partial x \partial y}\right\}\right] \\
& +\frac{E h}{2(1+\nu)}\left\{\frac{\partial^{2} u_{y}}{\partial x^{2}}+\frac{\partial^{2} u_{x}}{\partial x \partial y}+\frac{\partial^{2} u_{z}}{\partial x \partial y} \frac{\partial u_{z}}{\partial x}+\frac{\partial u_{z}}{\partial y} \frac{\partial^{2} u_{z}}{\partial x^{2}}\right\} \\
& =f_{y}\left(u_{x}, u_{y}, u_{z}\right)=0 \\
& -D\left(\frac{\partial^{4} u_{z}}{\partial x^{4}}+\nu \frac{\partial^{4} u_{z}}{\partial x^{2} \partial y^{2}}\right)-2 D(1-\nu) \frac{\partial^{4} u_{z}}{\partial x^{2} \partial y^{2}}-D\left(\frac{\partial^{4} u_{z}}{\partial y^{4}}+\nu \frac{\partial^{4} u_{z}}{\partial x^{2} \partial y^{2}}\right) \\
& +\frac{E h}{1-\nu^{2}}\left\{\frac{\partial^{2} u_{x}}{\partial x^{2}}+\frac{\partial^{2} u_{z}}{\partial x^{2}}+\nu\left(\frac{\partial^{2} u_{x}}{\partial x \partial y}+\frac{\partial u_{z}}{\partial y} \frac{\partial^{2} u_{z}}{\partial x \partial y}\right)\right\} \frac{\partial u_{z}}{\partial x} \\
& +\frac{E h}{2(1+\nu)}\left(\frac{\partial^{2} u_{x}}{\partial x \partial y}+\frac{\partial^{2} u_{y}}{\partial x^{2}}+\frac{\partial^{2} u_{z}}{\partial x^{2}} \frac{\partial u_{z}}{\partial y}+\frac{\partial u_{z}}{\partial x} \frac{\partial^{2} u_{z}}{\partial x \partial y}\right) \frac{\partial u_{z}}{\partial y} \\
& +\frac{E h}{1-\nu^{2}}\left\{\frac{\partial^{2} u_{x}}{\partial y^{2}}+\frac{\partial u_{z}}{\partial y} \frac{\partial^{2} u_{z}}{\partial y^{2}}+\nu\left(\frac{\partial^{2} u_{x}}{\partial x \partial y}+\frac{\partial^{2} u_{z}}{\partial x \partial y}\right)\right\} \frac{\partial u_{z}}{\partial y} \\
& +\frac{E h}{2(1+\nu)}\left(\frac{\partial^{2} u_{x}}{\partial y^{2}}+\frac{\partial^{2} u_{y}}{\partial x \partial y}+\frac{\partial^{2} u_{z}}{\partial x \partial y} \frac{\partial u_{z}}{\partial y}+\frac{\partial u_{z}}{\partial x} \frac{\partial^{2} u_{z}}{\partial y^{2}}\right) \frac{\partial u_{z}}{\partial x} \\
& =f_{z}\left(u_{x}, u_{y}, u_{z}\right)=0
\end{aligned}
$$

where $D=E h^{3} / 12\left(1-\nu^{2}\right)$ is membrane flexural rigidity. The above equations are grouped as:

$$
\boldsymbol{f}(\boldsymbol{u}(\boldsymbol{x}))=\left[\begin{array}{c}
f_{x}\left(u_{x}(x, y, z), u_{y}(x, y, z), u_{z}(x, y, z)\right) \\
f_{y}\left(u_{x}(x, y, z), u_{y}(x, y, z), u_{z}(x, y, z)\right) \\
f_{z}\left(u_{x}(x, y, z), u_{y}(x, y, z), u_{z}(x, y, z)\right)
\end{array}\right]=\mathbf{0}
$$


The boundary conditions for the top and bottom edges of the membrane are:

$$
\begin{array}{rlrl}
u_{x}\left(x,-\frac{b}{2}, 0\right) & =0 & u_{y}\left(x,-\frac{b}{2}, 0\right)=0 \\
u_{z}\left(x, \pm \frac{b}{2}, 0\right) & =0 & \left(-\frac{a}{2} \leq x \leq \frac{a}{2}\right) \\
\frac{\partial u_{z}}{\partial x}\left(x, \pm \frac{b}{2}, 0\right)=0 & \frac{\partial u_{z}}{\partial y}\left(x, \pm \frac{b}{2}, 0\right)=0 \\
\frac{\partial u_{z}}{\partial z}\left(x, \pm \frac{b}{2}, 0\right)=0 & \left(-\frac{a}{2} \leq x \leq \frac{a}{2}\right)
\end{array}
$$

The cyclic boundary condition for the left and right edges of the membrane is:

$$
\begin{array}{rlrl}
u_{x}\left(-\frac{a}{2}, y, 0\right) & =u_{x}\left(\frac{a}{2}, y, 0\right) & u_{y}\left(-\frac{a}{2}, y, 0\right) & =u_{y}\left(\frac{a}{2}, y, 0\right) \\
u_{z}\left(-\frac{a}{2}, y, 0\right) & =u_{z}\left(-\frac{a}{2}, y, 0\right) & \left(-\frac{1}{2} b \leq y \leq \frac{1}{2} b\right) \\
\frac{\partial u}{\partial x}\left(-\frac{a}{2}, y, 0\right) & =\frac{\partial u}{\partial x}\left(\frac{a}{2}, y, 0\right) & \frac{\partial u}{\partial y}\left(-\frac{a}{2}, y, 0\right)=\frac{\partial u}{\partial y}\left(\frac{a}{2}, y, 0\right) \\
\frac{\partial u}{\partial z}\left(-\frac{a}{2}, y, 0\right) & =\frac{\partial u}{\partial z}\left(\frac{a}{2}, y, 0\right) & \left(-\frac{1}{2} b \leq y \leq \frac{1}{2} b\right)
\end{array}
$$

While it is not possible to analytically solve these equations, they can be used to determine system symmetry and to explain membrane behavior. This topic is discussed in a later section.

\section{Finite element method (FEM) model}

The above geometry, properties and boundary conditions are modeled using ABAQUS, a finite element software package. We use the S4R shell elements provided by ABAQUS. For the mesh, equivalent divisions in $x$ and $y$ directions are constructed, resulting in $360 \times 120$ elements. The validity of this mesh density is discussed in Section V. It should be noted that only geometric nonlinearity is considered. Other factors such as material nonlinearity are not considered in the present study.

The static equilibrium of forces, i.e. nonlinear FEM equations based on the displacement method, are formally written as:

$$
\boldsymbol{F}(\boldsymbol{u}, \boldsymbol{f})=\mathbf{0}
$$


where $\boldsymbol{f}$ is the applied force for displacement $\boldsymbol{u}$. The static problem solves the equilibrium equation with $\delta u_{x}$ for the equilibrium point $(\boldsymbol{u}, \boldsymbol{f})$. The imposed displacement $\delta u_{x}$ is included in $\boldsymbol{u}$ and the constraint forces for displacement $\boldsymbol{u}$ are included in $\boldsymbol{f}$. Eq. (9) is a nonlinear equation in $\boldsymbol{u}$ and $\boldsymbol{f}$, which is difficult to solve. A solution method, e.g. an incremental calculation, is needed.

\section{Equilibrium path tracking method}

\section{A. Outline}

According to Senda, et al.[16], bifurcation points are bifurcation branching points and limit points[14]. When the solution passes through a limit point, snap-through bifurcation occurs and the equilibrium path jumps in a discontinuous manner. Branching bifurcation points are classified as the symmetric and the asymmetric bifurcation points. Symmetric and asymmetric bifurcation points generate symmetric and asymmetric bifurcation buckling, respectively. It is possible to predict the type of bifurcation structure using asymptotic theory. In the case of snap-through buckling and symmetric bifurcation buckling, the direction of the incremental displacement from the buckling point is equal to the eigenvector corresponding to a zero eigenvalue. For asymmetric bifurcation buckling, displacement and load should be changed to satisfy the relations of asymptotic theory discussed in Senda, et al.[16].

At bifurcation point $\left(\boldsymbol{u}_{c}, \boldsymbol{f}_{c}\right)$, the tangent stiffness matrix $\boldsymbol{K}$ has a zero eigenvalue $\lambda_{c}=0$ $(\operatorname{det} \boldsymbol{K}=0)$ and a corresponding eigenvector $\boldsymbol{\phi}_{c}$. In order to determine the position of the bifurcation point, a pinpointing procedure is similar to the method to solve the extended system described in Wriggers, et al.[15] is used. The eigenvalue analysis of the tangent stiffness matrix is performed and $\left(\boldsymbol{u}_{c}+\epsilon \boldsymbol{\phi}_{c}, \boldsymbol{f}_{c}\right)$ is computed. The perturbed state is in the eigenvector direction from the bifurcation point, where $\epsilon$ is an infinitesimal parameter. By assuming the perturbed state is near a post-bifurcation path, a convergence calculation, the Newton-Raphson iteration, is performed as described in Senda, et al.[16]. This static analysis searches for a new equilibrium point after the bifurcation. If a post-bifurcation equilibrium point cannot be found, the initial condition is changed by varying $\epsilon$ and the search for a new equilibrium point is repeated. 


\section{B. Analysis algorithm}

If there are multiple bifurcation points with multiple zero eigenvalues, an effective way to search for buckling modes is by linearly combining eigenvectors. An algorithm to search for a bifurcation solution, where the bifurcation point is a double singular point (i.e. $\boldsymbol{K}$ has two zero eigenvalues), is shown.

1. By increasing $\delta u_{x}$, an eigenvalue analysis of the tangent stiffness matrix $\boldsymbol{K}$ is performed.

2. Existence of the primary path is checked. For this purpose, path tracking without perturbation $\tilde{\boldsymbol{u}}$ is performed by increasing $\delta u_{x}$ from the initial point $\left(\boldsymbol{u}_{c}, \boldsymbol{f}_{c}\right)$.

3. The point $\left(\boldsymbol{u}_{c}+\boldsymbol{\epsilon}^{T} \boldsymbol{\Phi}, \boldsymbol{f}_{c}\right)$ is calculated where eigenvectors $\phi_{1}$ and $\phi_{2}$ correspond to the zero eigenvalues, $\boldsymbol{\Phi}=\left[\begin{array}{ll}\phi_{1} & \phi_{2}\end{array}\right], \boldsymbol{\epsilon}=[\epsilon \cos \theta \epsilon \sin \theta]^{T}$ and $0<\epsilon \ll 1,0 \leq \theta<2 \pi$. Vector $\boldsymbol{\epsilon}^{T} \boldsymbol{\Phi}$ is called the disturbance vector. A convergence calculation for the static analysis is performed from this state to obtain a new equilibrium point. Various values of $\theta$ are tested and then $\epsilon$ is increased gradually to seek the post-bifurcation path. If the solution is obtained, the path tracking is performed by increasing $\delta u_{x}$ from this state. If the solution is not found, $\epsilon$ or $\theta$ are changed and the search is repeated.

4. Point $\left(\boldsymbol{u}+\boldsymbol{\epsilon}^{T} \mathbf{\Phi}, \boldsymbol{f}\right)$ is calculated from the pre-buckling point $(\boldsymbol{u}, \boldsymbol{f})$, where $\boldsymbol{\epsilon}$ and $\boldsymbol{\Phi}$ are defined in step 3. If a solution is obtained, the path tracking is performed by decreasing $\delta u_{x}$ from this point.

An algorithm for a simple singular point follows the same progression but with a slight modification. In steps 3 and $4, \boldsymbol{u}_{c} \pm \epsilon \boldsymbol{\phi}$ is used instead of $\boldsymbol{u}_{c}+\boldsymbol{\epsilon}^{T} \boldsymbol{\Phi}$ where $\boldsymbol{\phi}$ is the eigenvector of the zero eigenvalue and $\epsilon$ is an infinitesimal parameter.

\section{Analysis methods of solution}

\section{A. Symmetric solutions}

Based on group theory[17], the equivalence of a system that possesses symmetry is discussed. The observed membrane has symmetry that is invariant with respect to $e, s, r$ and $s r$, where $e$ is the identity transformation, $s$ is the reflection transformation with respect to $x y$-plane, $r$ is the rotation 
transformation by $180^{\circ}$ about the $z$-axis and $s r$ is the combination of $s$ and $r$. This symmetry group is known as the dihedral group $D_{2}=\{e, r, s, s r\}$.

Deformation $\boldsymbol{u}_{0}(x, y, z)$ satisfies the governing equations (4) and the boundary conditions in Eqs. (5) to (6). Three deformations $\boldsymbol{u}_{s}(x, y, z), \boldsymbol{u}_{r}(x, y, z)$, and $\boldsymbol{u}_{s r}(x, y, z)$ are obtained from $\boldsymbol{u}_{0}(x, y, z)$ by means of the reflection transformation, the rotation transformation, and the rotation and reflection transformation, respectively. The three deformations $\boldsymbol{u}_{s}, \boldsymbol{u}_{r}$, and $\boldsymbol{u}_{s r}$ are the solutions satisfying the governing equations (4) and the boundary conditions in Eqs. (5) to (6).

Symmetry between two solutions is defined through an example. If deformation $\boldsymbol{u}_{1}(x, y, z)$ is equivalent to $\boldsymbol{u}_{s r}(x, y, z)$, there exists a symmetry where $\boldsymbol{u}_{0}$ agrees with $\boldsymbol{u}_{1}$ using the rotation and reflection transformation. Also, $\boldsymbol{u}_{1}$ is equivalent to $\boldsymbol{u}_{0}$ transformed by the rotation and reflection transformation. In other words, $\boldsymbol{u}_{0}$ and $\boldsymbol{u}_{1}$ have rotation and reflection symmetry. The deformation $\boldsymbol{u}_{1}$ is a different representation of $\boldsymbol{u}_{0}$.

Symmetry in a solution can also be defined through an example. If $\boldsymbol{u}_{0}(x, y, z)$ is equivalent to $\boldsymbol{u}_{r}(x, y, z)$, then $\boldsymbol{u}_{0}$ has symmetry that is invariant under the rotation transformation. Also, $\boldsymbol{u}_{0}$ has rotation symmetry. In this case, $\boldsymbol{u}_{r}$ is obtained, which is equivalent to $\boldsymbol{u}_{0}$ by the rotation transformation.

\section{B. Translation symmetry}

It is assumed that the solution $\boldsymbol{u}_{0}(x, y, z)$ satisfies the governing equation (4) and the boundary conditions in Eqs. (5) to (6). By translating $\boldsymbol{u}_{0}$ in the $x$-direction by $\Delta x$, a displacement $\boldsymbol{u}_{t}$ is obtained.

$$
\boldsymbol{u}_{t}(x, y, z)= \begin{cases}\boldsymbol{u}_{0}(x-\Delta x, y, z) & \left(-\frac{a}{2} \leq x-\Delta x \leq \frac{a}{2}\right) \\ \boldsymbol{u}_{0}(x-\Delta x+a, y, z) & \left(x-\Delta x<-\frac{a}{2}\right)\end{cases}
$$

Here, $\Delta x$ satisfies $0 \leq \Delta x<a$. The translation $\Delta x \pm n a$ where $(n=1,2, \ldots)$ can be considered as the translation $\Delta x$.

Because $\boldsymbol{u}_{0}(x, y, z)$ satisfies the governing equations, $\boldsymbol{f}\left(\boldsymbol{u}_{0}(x, y, z)\right)=\mathbf{0}$, the following holds 
true:

$$
\boldsymbol{f}\left(\boldsymbol{u}_{t}(x, y, z)\right)=\mathbf{0}
$$

Therefore, the displacement $\boldsymbol{u}_{t}(x, y, z)$ is also a solution to the governing equations. Displacement $\boldsymbol{u}_{t}(x, y, z)$ for $x \leq a / 2$ corresponds to $\boldsymbol{u}_{0}(x, y, z)$ for $x \leq a / 2-\Delta x$ because $\boldsymbol{u}_{t}$ is obtained by translating $\boldsymbol{u}_{0}$ in the $x$-direction by $\Delta x$. In turn, $\boldsymbol{u}_{t}(x, y, z)$ for $x \geq-a / 2$ corresponds to $\boldsymbol{u}_{0}(x, y, z)$ of $x \geq a / 2-\Delta x$. Therefore, the cyclic boundary conditions of $\boldsymbol{u}_{t}(x, y, z)$ at $x= \pm a / 2$ are satisfied naturally. From the above, it is clear that $\boldsymbol{u}_{t}(x, y, z)$, which is obtained by a translation of $\boldsymbol{u}_{0}(x, y, z)$ in the $x$-direction by $\Delta x$, also satisfies the governing equations and boundary conditions.

In addition, it can be said that the solution $\boldsymbol{u}_{t}(x, y, z)$ is a symmetric with respect to $\boldsymbol{u}_{0}(x, y, z)$ by translation $\Delta x$. In the following discussions, the two solutions are not considered as essentially different. In addition, $\Delta x$ can be any arbitrary value, resulting in an infinite number of solutions $\boldsymbol{u}_{t}(x, y, z)$ obtained by translation of $\boldsymbol{u}_{0}(x, y, z)$. These infinite number of solutions are identified by translational symmetry and are represented by only $\boldsymbol{u}_{0}$.

\section{Translation transformation calculation}

Since there is an infinite number of translations of a specific solution, the problem is to identify the value of the translation between the two solutions obtained by the analysis. The concept of phase correlation used in image processing[18] is useful for this purpose. The solution from an FE analysis is represented by node values resulting in a finite set of values whose size is $N_{x} \times N_{y}$. To determine the translation value between two finite sets, the phase correlation between the two sets is calculated as:

$$
\begin{aligned}
R(m, n) & =\frac{\hat{f}_{1}(m, n) \hat{f}_{2}^{*}(m, n)}{\left|\hat{f}_{1}(m, n) \hat{f}_{2}^{*}(m, n)\right|} \\
& =e^{i \frac{2 \pi}{N_{x}} m\left(\Delta n_{x}-1\right)} e^{i \frac{2 \pi}{N_{y}} n\left(\Delta n_{y}-1\right)}
\end{aligned}
$$

where $\hat{f}_{i}$ is the Fourier transformation of a finite set $f_{i}$ representing a solution $\boldsymbol{u}_{i}(x, y, z), \hat{f}_{2}^{*}$ indicates the complex conjugate of $\hat{f}_{2}$, and $\Delta n_{x}$ and $\Delta n_{y}$ are discrete translations in their re- 
spective directions. The phase correlation product $R(m, n)$ represents the inner product between individual frequencies that represent the two solutions. By calculating the inverse transformation $r\left(\Delta n_{x}, \Delta n_{y}\right)=\mathscr{F}^{-1}\{R(m, n)\}$, a function represented in the $\left(\Delta n_{x}, \Delta n_{y}\right)$ domain is formed. The value of this function indicates the inner products of two solutions for that specific translation.

The cyclic boundary conditions allow movement in only the $x$-direction, therefore $\Delta n_{y} \equiv 0$. This results in a one-dimensional problem to find the highest value of $r$ for $\Delta n_{x}$ corresponding to the largest degree of similarity.

Considering the original discrete solution, the resolution, the minimum value of the above described translational displacement is $a / N_{x}$. However, the solutions representing deformation are continuous. For the continuous solutions, the transversal displacement that results in best matches is not discrete. In order to obtain a higher resolution, the following calculation is performed. Using the FEM nodal displacements, a continuous function representing the deformation is constructed as a Fourier series. From the continuous function, a new set of discrete values is constructed by sampling of sub-node positions. By increasing the number of points considered as $M_{x}$, where $M_{x}>N_{x}$, the resolution of the translation increases. In the $y$ direction the resolution is unchanged because the translation is always 0 .

\section{Similarity value}

In order to identify similarity between the two solutions, the similarity value is constructed as the inner product of the solutions:

$$
<f_{1}\left(n_{x}, n_{y}\right), f_{2}\left(n_{x}, n_{y}\right)>=\sum_{p=1}^{N_{x}} \sum_{q=1}^{N_{y}} \hat{f}_{1}(m, n) \hat{f}_{2}^{*}(m, n)
$$

where $f_{j}\left(n_{x}, n_{y}\right)$ is a vector whose components are the displacement values sampled at all finite element nodes and $\hat{f}_{j}(m, n)$ is the discrete Fourier transformation of $f_{j}\left(n_{x}, n_{y}\right)$.

The similarity value is evaluated using the first bifurcation point of the membrane. Because of membrane symmetry, the first bifurcation point is also symmetric, as is theoretically predicted. Therefore, the theoretical similarity value between two deformations should be 1 . However, the above defined similarity value is based on a discrete set. When the similarity value of $f_{1}$ and $f_{2}$ are 
obtained numerically after the first bifurcation point, a value of $0.999995 \ldots$ is obtained. Therefore, the values after the fifth digit are considered as numerical error. During comparison of different deformations, the number of digits to which similarity is obtained is referred to as, e.g. 0.99999345 achieving similarity to within 5 digits.

The similarity value analysis uses the following procedure. The translation value between two solutions is obtained by phase correlation. One solution is translation transformed with the determined translation value. The similarity value is evaluated between the translated solution and the other solution.

\section{E. Bifurcation diagram}

A method of expressing the equilibrium path as it passes through the bifurcation point is needed. Here, the inner product of the eigenvector and the incremental displacement vector is used to make a bifurcation diagram.

At the bifurcation point $\left(\boldsymbol{u}_{c}, \boldsymbol{F}_{c}\right)$, the tangent stiffness matrix $\boldsymbol{K}_{c}$ becomes det $\boldsymbol{K}_{c}=0$. At this point there exist $N_{c}$ zero eigenvalues $\lambda_{c i}=0\left(i=1, \ldots, N_{c}\right)$ and corresponding eigenvectors $\phi_{c i}$.

$$
\boldsymbol{K}_{c i} \phi_{c i}=\lambda_{c i} \phi_{c i} \quad\left(i=1,2, \ldots, N_{c}\right)
$$

where $\phi_{c i}$ is normalized as $\left\|\phi_{c i}\right\|=1$. Using $\lambda_{c i}=0$, where $c_{i}$ is an arbitrary constant results in:

$$
\boldsymbol{K}_{c}\left(\sum_{i=1}^{N_{c}} c_{i} \boldsymbol{\phi}_{c i}\right)=\mathbf{0}
$$

As explained in the earlier section the incremental displacement $\tilde{\boldsymbol{u}}_{c}$ occurs whereas the incremental $\operatorname{load} \tilde{\boldsymbol{f}} \simeq \mathbf{0}$ :

$$
\boldsymbol{K}_{c} \tilde{\boldsymbol{u}}_{c} \simeq \mathbf{0}
$$


Combining Eq. (15) and Eq. (16), the following holds:

$$
\tilde{\boldsymbol{u}}_{c} \simeq \sum_{i=1}^{N_{c}} c_{i} \boldsymbol{\phi}_{c i}
$$

The displacement increment after the bifurcation point, $\tilde{\boldsymbol{u}}$ is expressed as a linear combination of eigenvectors $\phi_{c i}$ corresponding to the zero eigenvalues of the tangent stiffness matrix. The calculation of $c_{i}$ is:

$$
c_{i}=\tilde{\boldsymbol{u}}_{c} \cdot \phi_{c i}\left(i=1, \ldots, N_{c}\right)
$$

For construction of the bifurcation diagram, $c_{i}$ will be used.

\section{Numerical results}

\section{A. Validity of the FEM modeling}

Experimental confirmation of behavior of flat membranes with cyclic boundaries is not possible. However, experimental data are available for a flat membrane model where cyclic boundaries are replaced with free boundaries[4]. Therefore, the geometry, membrane properties, and boundary conditions mentioned in Section II are compared and FEM model accuracy is tested. The numerical accuracy of the free boundary model is discussed in detail in Senda, et al[16].

The free boundary membrane was modeled using a 4 node shell element with reduced Gauss integration. By increasing the shear displacement, the wavelength and amplitude of wrinkles were observed as indicators of wrinkle shape. By analyzing models with different numbers of elements, it was concluded that $360 \times 120=4.23 \times 10^{4}$ elements achieve sufficient convergence.

To obtain the same level of convergence in the cyclic boundary and cylindrical membrane models, convergence of wrinkle wavelength and amplitude for a single wrinkle was observed. In the case of the free boundary membrane, the free boundaries distort the shape of the wrinkles. Therefore, six wrinkles from each free boundary were omitted. The remaining wrinkles in the middle of the membrane mostly have uniform shapes, similar to those of the cyclic boundary membrane. For the remaining wrinkles, converged values are established if a wrinkle has 8 or more elements along the 

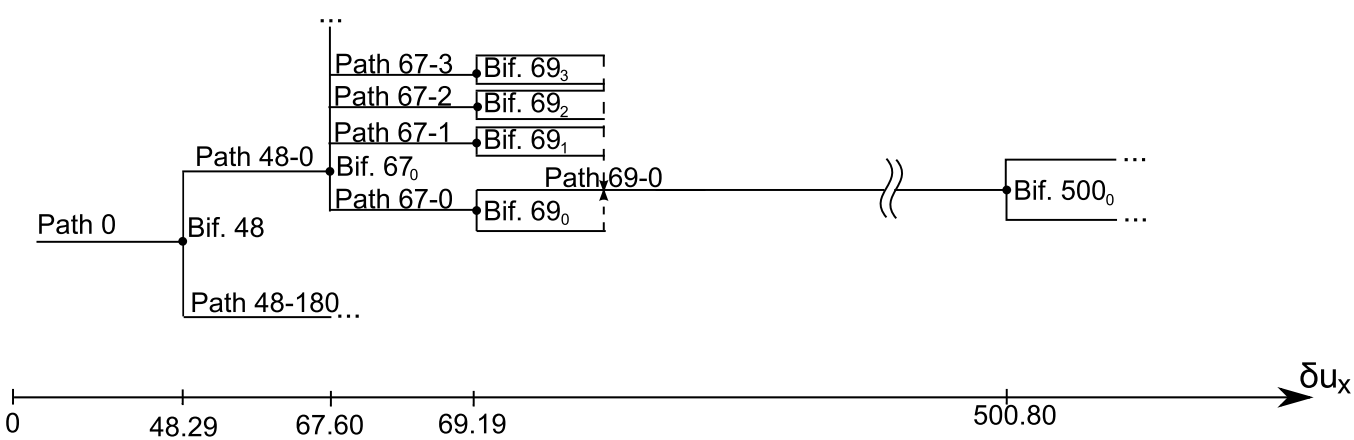

Fig. 3 Flat membrane schematic bifurcation diagram

$x$-direction. This criterion was adopted for the cyclic boundary and cylindrical membrane models. Therefore, the base number of elements for the flat membrane with cyclic boundaries and for the cylindrical membrane is selected as $390 \times 130$. Additionally, more elements, e.g. $408 \times 136,432 \times 144$, and $600 \times 200$ were also used for analysis. The results from these numbers of elements were compared to the result from $390 \times 130$ elements to confirm that the results converged and were insensitive above $5.07 \times 10^{4}$ elements.

The convergence of the numerical results is controlled by two additional parameters in the ABAQUS calculation. First, $R_{n}^{\alpha}$ is the convergence criterion for the ratio of the largest residual to the corresponding average flux norm. The default value is $R_{n}^{\alpha}=5 \times 10^{-3}$. Second is $C_{n}^{\alpha}$, which is the convergence criterion for the ratio of the largest solution correction to the largest corresponding incremental solution value. Its default value is $C_{n}^{\alpha}=1 \times 10^{-2}$. For general analysis, the default values are sufficient; however, to obtain more precise results, values that are $10^{-4}$ times of the default values are used combined with the $\delta u_{x}$ increment of $10^{-7}[\mathrm{~m}]$.

\section{B. Flat membrane behavior}

In this section, the behavior of the cyclic boundary membranes will be shown. The schematic bifurcation diagram is shown in Fig. 3. The diagram shows the positioning of bifurcation points and paths that lead to and from the bifurcation points. For notation, bifurcation points are indicated by 'Bif. $X_{n}$ ', where $X$ indicates the value of the $\delta u_{x}$ at the point of bifurcation. The subscript $n$ is an identifier used if there are multiple bifurcation points at the same value of $\delta u_{x}$. Paths are indicated by 'Path $Y$-m', where $Y$ is the value of the $\delta u_{x}$ where the path starts, and $m$ is an 
identifier indicating the value of $\theta$ for which the path was obtained, as discussed in section IIIB.

Because of membrane symmetry, only a small part of the total bifurcation structure is shown. The translation symmetry is achieved for any arbitrary value of translation, resulting in an infinite number of paths representing a single unique wrinkle pattern. Therefore, only the original path is drawn. The analysis of the membrane shows the behavior for $0 \leq \delta u_{x} \leq 510.00 \times 10^{-6}[\mathrm{~m}]$.

\section{Bif. 48}

The initial condition is a flat membrane. First, tension $\delta u_{y}=30 \times 10^{-6}[\mathrm{~m}]$ is applied in the $y$-direction and maintained constant throughout the analysis. Then, increasing $\delta u_{x}$ results in a tensile first principal stress and a compressive second principal stress. Once the second principal stress reaches a critical value, the first bifurcation occurs.

The first bifurcation occurs at $\delta u_{x}=48.29 \times 10^{-6}[\mathrm{~m}]$ as Bif. 48. This bifurcation generates 32 wrinkles, i.e. 16 upward convex and 16 downward convex wrinkles. Out-of-plane deformation for the membrane is shown in Fig. 6 a). Figure 7 a) shows the out-of-plane deformation in section BB'. As indicated by the figures, all wrinkles have the same shape. As a result, the deformation that is translated by an integer number of wrinkle wavelengths is equivalent to the original deformation. Hence, this deformation has symmetry that is invariant under the translation transformation by $n a / 16(n= \pm 1, \pm 2, \ldots)$ in the $x$-direction.

The bifurcation structure of Bif. 48 is shown in Fig. 8 a). The structure is a symmetric bifurcation. As a result, wrinkles rapidly increase in amplitude from a flat membrane after the bifurcation. The paths are calculated by considering $\theta$ at increments of $30^{\circ}$. However, a path can be obtained for any $\theta$. The disturbance vector is constructed from two orthogonal eigenvectors. Therefore, the disturbance vector of $\theta=0^{\circ}$ is translated in $x$-direction in accordance with $\theta$. Hence, a path obtained for a certain $\theta$ represents a path that is obtained from the path of $\theta=0^{\circ}$ by translating in $x$-direction.

There are Path 48-0 and Path 48-180 after Bif. 48 in Fig. 3. Because this is a symmetric bifurcation point, the deformation patterns of Path 48-0 and Path 48-180 have reflection symmetry. There is only one wrinkle pattern after Bif. 48. A single path, Path $48-0$, defined by $\theta=0^{\circ}$ is 
tracked by increasing $\delta u_{x}$.

\section{Bif. 67}

On Path 48-0, the membrane has 32 wrinkles with the same shape. Increasing $\delta u_{x}$ results in wrinkle amplitude augmentation. The second bifurcation occurs at $\delta u_{x}=67.60 \times 10^{-6}[\mathrm{~m}]$ as Bif. $67_{0}$. The out-of-plane deformation of the membrane is shown in Fig. 6 b). Figure 7 b) shows the out-of-plane deformation in section BB'. For Path 48-0, the deformation has a sinusoidal shape with a period of $a / 16$ in the $x$-direction and a mean of zero. After the bifurcation, a variation of the mean in the form of a sinusoidal deformation with a period of $a / 2$ in the $x$-direction is observed in section BB'. While the variation is observed near the fixed boundaries, it is not observed in the middle of the membrane in section AA'.

The bifurcation structure for Bif. 67 is shown in Fig. 8 b). Paths are obtained for values of $\theta$ from $0^{\circ}$ to $360^{\circ}$ for every $22.5^{\circ}$. Figure $8 \mathrm{c}$ ) shows paths for $\theta$ from $0^{\circ}$ to $22.5^{\circ}$.

A single group is defined for $0^{\circ} \leq \theta \leq 22.5^{\circ}$. This is due to the sinusoidal deformation of period $a / 2$ being imposed onto the base sinusoidal deformation of period $a / 16$. The value of $\theta$ defines the $x$-direction position of the $a / 2$ period sinusoidal shape. Considering that the full range of imposition of $\theta$ is $360^{\circ}$, the period of $a / 16$ in $x$ defines the range of a group as $360^{\circ} / 16=22.5^{\circ}$.

For a given $\theta$, an equilibrium path will be produced where the peaks of the $a / 2$ period sinusoidal deformation produce two wrinkles with larger amplitude values. As indicated by Fig. 3, there are many paths leading from Bif. $67_{0}$. However, the paths do not exist only for discrete values of $\theta$ as the figure suggests, but for any arbitrary value. The result is an infinite number of paths generated at Bif. $67_{0}$. Any two paths in a group have different deformation shapes when their $\theta$ values are not the same.

By considering a path in group 1 defined as $\theta=\theta^{\prime}$, any group $n$ path defined as $\theta=\theta^{\prime}+n \times 22.5^{\circ}$ will have a 5 digit similarity with the group 1 path after a translation transformation by $n a / 16$. This means that groups represent sets of deformations that are equivalent by translation symmetry. Therefore, only paths of one group will be tracked until the next bifurcation. 
3. Bif. 69

On any Path 67, the membrane has 32 wrinkles where two wrinkles have slightly larger amplitudes. By increasing $\delta u_{x}$, the wrinkle amplitude increases. The third bifurcation occurs at $\delta u_{x}=69.19 \times 10^{-6}[\mathrm{~m}]$ as Bif. 69 . This is a symmetric snap through bifurcation. The out-of-plane deformation after this bifurcation is shown in Fig. 6 c). Figure 7 c) shows the deformation in section BB'. The number of wrinkles increases by 2 resulting in a 34 wrinkle pattern. All wrinkles in this pattern have the same shape. As a result, this deformation has symmetry that is invariant under the translation transformation of $n a / 16(n= \pm 1, \pm 2, \ldots)$ in the $x$-direction.

The bifurcation structure of Bif. 69 is shown in Fig. 8 d). There are two paths leading from the bifurcation point. Wrinkles are generated from an unstable split of one of the existing wrinkles. Two wrinkles are candidates for splitting. They correspond to the two wrinkles with slightly larger amplitudes. Therefore, each of the paths after bifurcation represent one of the wrinkles that splits.

Comparison of all paths generated at Bif. 69, based upon all paths of a single group from Bif. 67, results in 5 digit similarity. Also, the comparison of individual wrinkles of a wrinkle pattern shows that all wrinkles are the same. Therefore, the conclusion is that after Bif. 69 there exists a single unique wrinkle pattern and an infinite number of equilibrium paths. All these paths correspond to translation transformations in the $x$-direction of the unique wrinkle pattern. Because of this, only one path is selected for tracking.

\section{Path 69-0}

Path 69-0 is the selected path after Bif. 69 and it contains 34 wrinkles that have the same shape. The following observed behavior occurs at regular equilibrium points. The tangent stiffness matrix remains positive definite until the next bifurcation.

Path $69-0$ is observed for $69.19 \times 10^{-6} \leq \delta u_{x} \leq 500.80 \times 10^{-6}[\mathrm{~m}]$. By increasing $\delta u_{x}$, collapsed sections are generated near the fixed boundaries on existing wrinkles. Figure $6 \mathrm{~d}$ ) shows the out-ofplane deformation at $\delta u_{x}=85.00 \times 10^{-6}[\mathrm{~m}]$. Figure $7 \mathrm{~d}$ ) shows the deformation in section BB'. The collapsed sections appear as local decreases in the wrinkle amplitude near the fixed boundaries.

By increasing $\delta u_{x}$, these sections grow in size and number. Figures 6 e) and 7 e) show the 
collapsed section at $\delta u_{x}=500.00 \times 10^{-6}[\mathrm{~m}]$. The existence of these collapsed sections resulted in all wrinkles having different shapes, where previously all wrinkles were uniform.

\section{Bif. 500}

The later part of Path 69-0 is a membrane that contains collapsed sections on existing wrinkles. The fourth bifurcation occurs at $\delta u_{x}=500.80 \times 10^{-6}[\mathrm{~m}]$ as Bif. 500. This bifurcation is a snap through bifurcation where three wrinkles split and generate six new wrinkles. The out-of-plane deformation is shown in Fig. $6 \mathrm{f}$ ). Figure $7 \mathrm{f}$ ) shows the deformation in section BB'. The result of the bifurcation is a 40 wrinkle pattern.

The process of wrinkle generation can be observed in Fig. 9. The membrane starts as in Fig. 9 a). The largest collapsed sections are destabilized. A collapsed section will rapidly expand along the length of the wrinkle until it meets with the opposite collapsed section in the middle. The affected wrinkles start to split as in Fig. 9 c). The split is then finalized as in Fig. 9 d). The collapsed sections on the other wrinkles remain.

It can be concluded that after $\delta u_{x} \approx 80 \times 10^{-6}[\mathrm{~m}]$, the behavior of the cyclic boundary membrane is summarized as follows. Collapsed sections are generated near the fixed boundaries, and as shear deformation is increased, they increase in size. At a specific value of shear deformation, they destabilize the large wrinkle and split it in half.

\section{Cylindrical membrane behavior}

In this section, the behavior of the cylindrical membrane is presented. The schematic bifurcation diagram is shown in Fig. 4. The diagram shows the positioning of bifurcation points and paths that lead to and from those bifurcation points. The analysis was performed for $0 \leq \delta u_{x} \leq 2180.00 \times$ $10^{-6}[\mathrm{~m}]$. The analysis process is the same as for the flat membrane. By comparing Fig. 4 with Fig. 3, it can be concluded that the bifurcation points are more spread out and there are fewer equilibrium paths. 

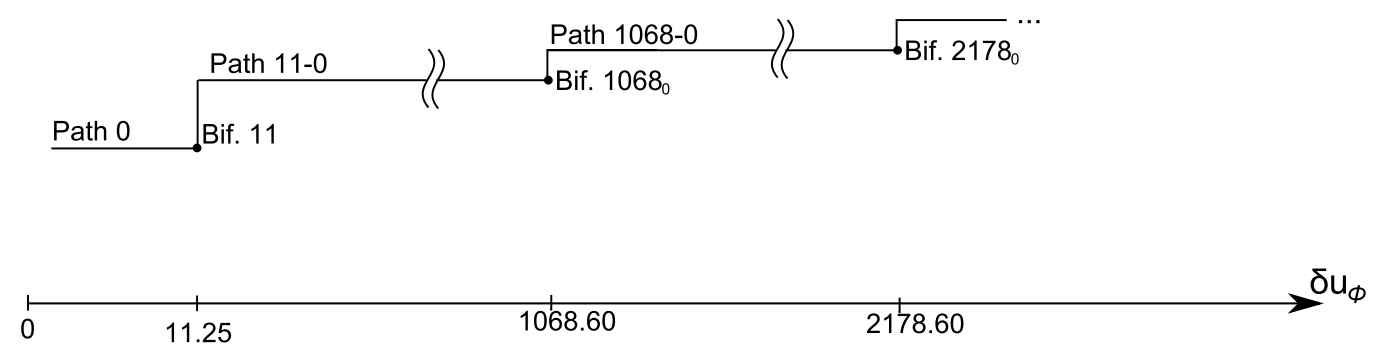

Fig. 4 Cylindrical membrane schematic bifurcation diagram

\section{Bif. 11}

The initial condition is a cylindrically shaped membrane where every point on the mid-plane is located on the $(x, y)$ plane at $z=0$. In this state, the displacement normal to the membrane surface is $u_{z}(x, y)=0$. An initial tension $\delta u_{y}=30 \times 10^{-6}[\mathrm{~m}]$ is applied in the $y$-direction and maintained constant throughout the analysis. This results in tension in both $x$ and $y$-directions. The tension in $y$-direction results in a small reduction in radius halfway between the top and bottom of the membrane. The fixed boundaries, maintain the original radius of the cylinder.

By applying the shear displacement $\delta u_{x}$, the radius continues to decrease due to an increase in stress. The first bifurcation point occurs at $\delta u_{x}=11.25 \times 10^{-6}[\mathrm{~m}]$ as Bif. 11. This bifurcation generates the initial 46 wrinkles, i.e. 23 upward convex and 23 downward convex wrinkles. The out-of-plane deformation $u_{z}$ is shown in Fig. 10 a). Figure 11 a) shows the out-of-plane deformation in section BB'. As a result, the deformation that is translated by an integer number of the wrinkle wavelength is equivalent to the original deformation. Hence, the symmetry of this deformation pattern is invariant under the $x$ translation transformation of $n a / 23(n= \pm 1, \pm 2, \ldots)$.

The bifurcation point is a double singular point. A post-bifurcation path can be obtained for any $\theta$, where different values of $\theta$ correspond to the same wrinkle pattern translated in the $x$-direction. Because of this symmetry, only a single path is tracked.

\section{Path 11-0}

The initial state is a cylindrical membrane with 46 wrinkles. The following behavior occurs at regular equilibrium points. The tangent stiffness matrix remains positive definite until the next bifurcation. 
Path 11-0 occurs for $11.25 \times 10^{-6} \leq \delta u_{x} \leq 1068.60 \times 10^{-6}[\mathrm{~m}]$. Initially, the increased shear displacement results in increased out-of-plane displacement. At $\delta u_{x}=432.00 \times 10^{-6}[\mathrm{~m}]$, collapsed sections are generated on only upward convex wrinkles near the fixed boundaries. The out-of-plane deformation is shown in Fig. $10 \mathrm{~b}$ ).

Figure $11 \mathrm{~b}$ ) shows the out-of-plane deformation in section BB' at $\delta u_{x}=432.00 \times 10^{-6}[\mathrm{~m}]$. After Bif. 11, both upward convex and downward convex wrinkles have similar shapes. The increasing shear results in negative $z$-direction displacement for all points on the membrane because of its cylindrical shape. The increasing compression results in positive $z$-direction displacements for upward convex wrinkles and negative $z$-direction displacement for downward convex wrinkles. In total, all points on the membrane, aside from those at fixed boundaries, are displaced in the negative $z$-direction at varying rates. Because of this, the curvature of upward convex wrinkles decreases, while the curvature of downward convex wrinkles increases.

Initially, collapsed sections are very small and slowly increase in size as the shear displacement increases. At $\delta u_{x}=1068.00 \times 10^{-6}[\mathrm{~m}]$ they are clearly visible as shown in Figs. $\left.10 \mathrm{c}\right)$ and $\left.11 \mathrm{c}\right)$.

For the length of the equilibrium path, these collapsed sections maintain a fixed position on upward convex wrinkles. As a result, the number of collapsed sections is also fixed. Because of this, all upward convex wrinkles maintain the same shape, and translational symmetry of the shape is maintained also. This deformation has symmetry that is invariant under the $x$-translation transformation of $n a / 23(n= \pm 1, \pm 2, \ldots)$ in $x$-direction.

\section{Bif. 1068}

The membrane state before the bifurcation is a 46 wrinkle pattern where every upward convex wrinkle has collapsed sections. The bifurcation point occurs at $\delta u_{x}=1068.60 \times 10^{-6}[\mathrm{~m}]$ as Bif. 1068 . The point is a snap through bifurcation that is a result of collapsed sections reaching a critical size. This bifurcation point is a double singular point. From 23 wrinkles with collapsed sections, 4 wrinkles are selected and split in half. The selected wrinkles depend on the value of $\theta$. The outof-plane deformation after the split is shown in Fig. $10 \mathrm{~d}$ ). Figure $11 \mathrm{~d}$ ) shows the out-of-plane deformation in section BB'. The results are 8 new wrinkles and a 54 wrinkle pattern. 
The process of wrinkle splitting can be observed in Fig. 12. The collapsed sections expand in the longitudinal direction of a upward convex wrinkle. The out-of-plane displacement of the wrinkle increases in the negative $z$-direction and the two collapsed sections join. The increase in the out-ofplane displacement continues until the downward convex wrinkle in the middle has the same shape as the remaining downward convex wrinkles. During the process, the wavelength of the original wrinkles is reduced to fit the new wrinkles.

The resulting pattern has 54 wrinkles where all upward convex wrinkles have the same shape, and all downward convex wrinkles have the same shape. While different values of $\theta$ select different wrinkles for splitting, the final wrinkle pattern is always the same. This deformation has symmetry that is invariant under the translation transformation by $n a / 27(n= \pm 1, \pm 2, \ldots)$ in the $x$-direction. Only Path 1068-0 is tracked because there is only one unique wrinkle pattern.

\section{Bif. 2178}

Path tracking of Path 1068-0 shows no new behavior. The increasing shear displacement results in increased out-of-plane displacement. As a result, the wrinkle pattern just before Bif. 2178 is a 54 wrinkle pattern, where 27 upward convex wrinkles have collapsed sections. The out-of-plane deformation is shown in Fig. 11 e). Figure 11 e) shows the out-of-plane deformation in section BB'.

The bifurcation point occurs at $\delta u_{x}=2178.60 \times 10^{-6}[\mathrm{~m}]$ as Bif. 2178. Similar to Bif. 1067, this point is a snap through bifurcation as a result of collapsed sections reaching critical size. However, at this point 7 wrinkles have split and result in a 68 wrinkle pattern. The new wrinkle pattern is shown in Fig. $11 \mathrm{f})$.

The process of wrinkle generation is the same as at Bif. 1068. Depending on the value of $\theta$, the 7 collapsed sections are selected and expanded in the longitudinal direction of a upward convex wrinkle. After the collapsed sections join, new wrinkles grow until they become the same size as the remaining wrinkles.

Because the behavior from the previous bifurcation point is repeated, the increase in shear is stopped at this point. It can be concluded that the same behavior, where collapsed sections split existing wrinkles, is repeated. 


\section{Generation of collapsed sections}

As shown in the results, there exist stable equilibrium paths without bifurcation points, where small wrinkles or collapsed sections are generated. To understand this behavior, the wrinkle geometry and membrane equilibrium are observed near the fixed boundaries. The geometry of a

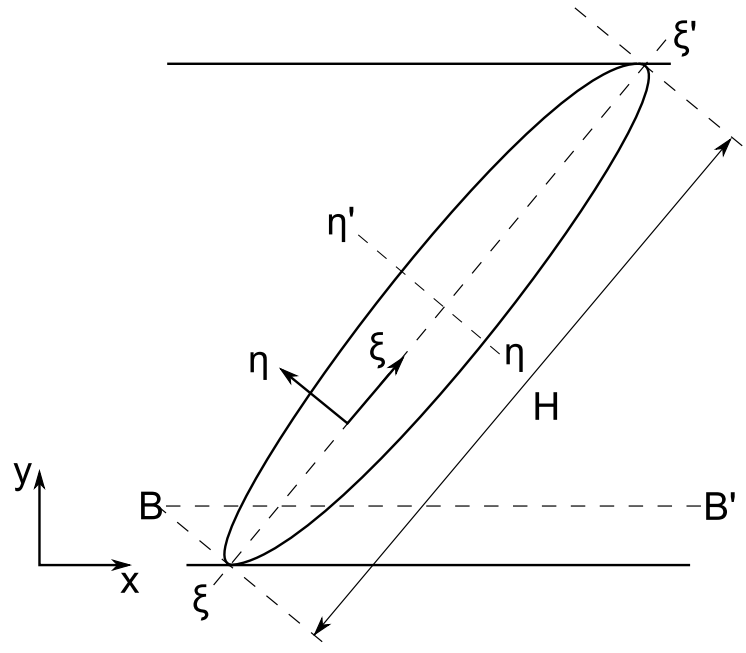

Fig. 5 Wrinkle geometry.

single wrinkle in the flat membrane is shown in Fig. 5. The same figure applies to the cylindrical membrane. Because in the cylindrical membrane collapsed sections occur only on upward convex wrinkles, the following discussion applies to an upward convex wrinkle for the flat membrane as well as the cylindrical one. The wrinkle discussed is indicated by the $\xi \xi^{\prime}$ section in Figs. 6 d) and 10 b).

\section{Flat membrane}

After Bif. 69, the wrinkle pattern consists of 34 sinusoidal wrinkles, as shown in Fig. 6 c). The first principal stress, $\sigma_{\xi}$, is shown in Fig. 13 a). The second principal stress, $\sigma_{\eta}$, is shown in Fig. 13 b). The stress state for the entire membrane is uniaxial compression. The largest first principal stress, $\sigma_{\xi}$, is in the middle of the membrane but falls off slightly toward the fixed boundaries. The second principal stress, $\sigma_{\eta}$, is smallest near the fixed boundaries but increases toward the middle of the membrane.

The interval $74.0 \times 10^{-6} \leq \delta u_{x} \leq 82.0 \times 10^{-6}[\mathrm{~m}]$ is observed for sections $\xi \xi^{\prime}$ and $\eta \eta^{\prime}$. The position on the section is defined by node values based on the finite element nodes. Section $\xi \xi^{\prime}$ 
starts at the bottom boundary and finishes at the top. The sections cross each other at node 15 of section $\xi \xi^{\prime}$, and node 9 of section $\eta \eta^{\prime}$. Section $\eta \eta^{\prime}$ observes 8 nodes on both side of section $\xi \xi^{\prime}$, for a total length of 16 nodes.

Figures $15 \mathrm{a}$ ) and b) show the principal stress values on section $\xi \xi^{\prime}$. Figure $15 \mathrm{c}$ ) shows the out-of-plane displacement, $u_{z}$, on section $\xi \xi^{\prime}$. Figure $15 \mathrm{~d}$ ) shows the curvature, $\kappa_{\eta}=\partial^{2} u_{z} / \partial \xi^{2}$, on section $\xi \xi^{\prime}$. Figure 15 e) shows the out-of-plane displacement, $u_{z}$, on section $\eta \eta^{\prime}$. Figure $\left.15 \mathrm{f}\right)$ shows the curvature, $\kappa_{\xi}=\partial^{2} u_{z} / \partial \eta^{2}$, on section $\eta \eta^{\prime}$. As $\delta u_{x}$ increases, out-of-plane displacement up to node 30 decreases. As a result, the region of positive curvature $\kappa_{\eta}$ expands from the fixed edge toward the middle of the membrane. At $\delta u_{x}=76.0 \times 10^{-6}[\mathrm{~m}]$, the decrease in wrinkle amplitude reached the point where the wrinkle becomes flat in the $\eta$-direction, as seen in Figs. 15 e) and f). At the flat top of the wrinkle, both $\kappa_{\eta}$ and $\kappa_{\xi}$ are observed as almost 0 . The amplitude drops further and a upward convex wrinkle gains a downward convex peak. At this point both curvature $\kappa_{\eta}$ and $\kappa_{\xi}$ change their signs. The result is a collapsed section.

\section{Cylindrical membrane}

The first principal stress, $\sigma_{\xi}$ is shown in Fig. 14 a). The second principal stress, $\sigma_{\eta}$ is shown in Fig. $14 \mathrm{~b}$ ). The stress state for the most of the membrane is uniaxial compression. Near the fixed boundaries, there are regions of biaxial tension. These regions correspond to the upward convex wrinkles. The first principal stress, $\sigma_{\xi}$, is largest in the middle of the membrane and falls off slightly toward the fixed boundaries. The second principal stress, $\sigma_{\eta}$, is largest at the fixed boundary. Its value changes rapidly from tension to compression by moving away from the fixed boundary. It finally converges to 0 in the middle of the membrane.

The interval $372.0 \times 10^{-6} \leq \delta u_{x} \leq 452.0 \times 10^{-6}[\mathrm{~m}]$ is observed in sections $\xi \xi^{\prime}$. The position on the section is defined by node values based on the finite element nodes. Section $\xi \xi^{\prime}$ starts at the bottom boundary and finishes at the top boundary.

Figures 16 a) and b) show the principal stress values on section $\xi \xi^{\prime}$. Figures 16 c) and d) show the out-of-plane displacement, $u_{z}$, on section $\xi \xi^{\prime}$. Figure 16 e) shows the curvature, $\kappa_{\eta}$, on section $\xi \xi^{\prime}$. Figure $16 \mathrm{f}$ ) shows the curvature, $\kappa_{\xi}$, on section $\xi \xi^{\prime}$. As $\delta u_{x}$ increases, the out-of-plane 
displacement along section $\xi \xi^{\prime}$ decreases, but at different rates along the section. The result is a change in curvature along the $\xi \xi^{\prime}$ section. For $\delta u_{x}=412.0 \times 10^{-6}[\mathrm{~m}]$, the curvature $\kappa_{\xi}$ becomes 0 at node 4 . At the same point and shear value, the curvature $\kappa_{\eta}$ is also 0 , indicating that the wrinkle has become flat. Further increase in shear displacement results in the curvature value changing signs, and a upward convex wrinkle gains a downward convex region. Therefore, a collapsed section is generated.

\section{Generation mechanism of collapsed sections}

Following the discussion about thin plate bending in Timoshenko[3], the equilibrium of a plate element in the $z$-direction can be written as

$$
\frac{\partial Q_{\xi}}{\partial \xi}+\frac{\partial Q_{\eta}}{\partial \eta}=N_{\xi} \kappa_{\eta}+N_{\eta} \kappa_{\xi}
$$

The left side of Eq. (19) represents the bending of the plate due to the transverse force $Q$, while the right side represents the membrane behavior due to mid-plane force $N$. The mid-plane forces $N_{\xi}$ and $N_{\eta}$ are obtained by integration of principal stress values over the membrane thickness. The values can be approximated as $N_{\xi}=h \sigma_{\xi}$ and $N_{\eta}=h \sigma_{\eta}$, where $\sigma_{\xi}$ and $\sigma_{\eta}$ are the first and second principal stress on the mid-plane, and $h$ is the membrane thickness. The transverse forces are shown in Fig. $15 \mathrm{~g}$ ) as $Q_{\xi}$ and in Fig. $15 \mathrm{~h}$ ) as $Q_{\eta}$ for the flat membrane. At the fixed boundary, the transverse forces correspond to the reaction forces from the boundary. By moving away from the fixed boundary, both $Q_{\xi}$ and $Q_{\eta}$ increase rapidly toward 0. By observing the equilibrium equation, it can be concluded that very near the fixed boundaries, the membrane behavior is determined by both transversal and mid-plane forces. In other words, the membrane behaves like a thin plate. By moving away from the fixed boundaries, the effects of transverse forces fall off and the behavior is dominated by mid-plane forces. Therefore, the left side of Eq. (19) is approximately zero, and the equation becomes a statement of membrane equilibrium.

For the flat membrane, near fixed boundaries, positive $\sigma_{\xi}$ over positive $\kappa_{\eta}$ results in an upward force. The negative $\sigma_{\eta}$ over negative $\kappa_{\xi}$ also results in an upward force. Equilibrium is maintained by downward transverse forces near the fixed boundaries, due to the bending stiffness of the mem- 
brane. Because of the bending stiffness and the boundary conditions, $\kappa_{\eta}$ is positive and out-of-plane displacement is inhibited

Collapsed sections occur in a region of positive $\sigma_{\xi}$ and negative $\sigma_{\eta}$. As shown in Fig. 15 a), there is only a small percentage difference in $\sigma_{\xi}$ between the values in the middle of the membrane and at collapsed sections, relative to the total value. By increasing $\delta u_{x}$, the length of the membrane along the $\xi$-direction increases, resulting in higher values of $\sigma_{\xi}$.

As shown in Fig. $15 \mathrm{~b}$ ), there is an order of one difference in $\sigma_{\eta}$ magnitude between the values in the middle of the membrane and near the fixed boundaries. Towards the middle of the membrane, the reduction in length along the $\eta$-direction does not occur because the wrinkle out-of-plane displacement increases which releases stress. Approaching the fixed boundaries, the increase in $\delta u_{x}$ results in the increase in compression, meaning there exists a reduction in length along the $\eta$-direction. This is because the fixed boundaries limit the increase in out-of-plane deformation and prevent the release of stress. This behavior is seen in Fig. 15 c). Because the stress cannot be released by increasing the out-of-plane deformation, the wrinkle number is increased instead. This increase in wrinkle number is represented by collapsed sections.

For the cylindrical membrane, the transverse forces are given in Fig. $16 \mathrm{~g})$ as $Q_{\xi}$ and in Fig. 16 h) as $Q_{\eta}$. Near the fixed boundaries, positive $\sigma_{\xi}$ over negative $\kappa_{\eta}$ results in a downward force. The positive $\sigma_{\eta}$ over negative $\kappa_{\xi}$ also results in a downward force. Due to the bending stiffness of the membrane, equilibrium is maintained by the upward transverse forces near the fixed boundaries.

Again, collapsed sections occur in a region of positive $\sigma_{\xi}$ and negative $\sigma_{\eta}$. As shown in Fig. 16 a), relative to the total value, there is only a small percentage of difference in $\sigma_{\xi}$ between the values in the middle of the membrane and the values at collapsed sections. By increasing $\delta u_{x}$, the length of the membrane along the $\xi$-direction increases, resulting in higher values of $\sigma_{\xi}$.

For $\sigma_{\eta}$ shown in Fig. $16 \mathrm{~b}$ ), there is an order of one difference in magnitude between the values in the middle of the membrane and those near the fixed boundaries. However, the region of uniaxial compression is at the edge of the biaxial tension region that exists near the fixed boundaries. Because of the proximity of the biaxial tension region, the amplitude of compression is smaller compared to the flat membrane. Towards the middle of the membrane, the reduction in length along the $\eta$ - 
direction does not occur because the wrinkle out-of-plane displacement is increased, which releases stress. At fixed boundaries, the constrained out-of-plane deformation prevents the release of stress. This behavior is seen in Fig. 16 c). Similar to the flat membrane, the stress cannot be released by increasing the out-of-plane deformation. Therefore, the wrinkle number is increased instead. This increase in wrinkle number is represented by collapsed sections.

From the above behavior, it can be concluded that the generation of collapsed sections is caused by fixed boundaries. These boundaries generate a stress state of uniaxial compression and constrain the wrinkle amplitude. For a typical wrinkle, the increasing shear results in an amplitude and frequency increase. Because the fixed boundaries limit the amplitude, the frequency increase is used to relieve membrane stress. The outcome is the generation of collapsed sections.

\section{Effects of the cylindrical shape}

While the mechanism for generating the collapsed sections is similar for both types of membranes, there are several important differences. As was shown in Section V B 4 for the flat membrane, collapsed sections change position and increase in number. However, the discussion about the cylindrical membrane in Section VC 2 showed the collapsed section position and number is fixed to the existing upward convex wrinkles. The result is a uniform wrinkle pattern in which all upward convex wrinkles are the same and all downward convex wrinkles are also the same.

By observing the principal stress value in both membranes, the first principal stress $\sigma_{\xi}$ has a similar distribution along the surface of the membrane. The second principal stress $\sigma_{\eta}$ also has a similar distribution in the middle section of the membrane. However near the fixed boundaries, $\sigma_{\eta}$ in the cylindrical membrane varies between tension and compression, while in the flat membrane it is constant compression along the boundary. These stress distributions are shown in Figs. 13 b) and $14 \mathrm{~b})$.

Initially, the membrane is stretched in the $y$-direction, before applying any shear. Therefore, the $y$-direction corresponds to the $\xi$-direction and the $x$-direction corresponds to the $\eta$-direction. The stretch results in biaxial tension for the entire membrane. This tension in the $\eta$-direction reduces the radius of the membrane. Starting from the fixed boundaries in the $\xi$-direction, the radius of the 
membrane decreases rapidly and becomes almost constant in the middle. The rapid reduction in radius means that the tensile $\sigma_{\eta}$ is much larger near the fixed boundaries than in the middle of the membrane, where it is almost zero. Therefore, a single region of tensile $\sigma_{\eta}$ spans the length of the membrane as shown in Fig. $17 \mathrm{~b}$ ). By adding shear, $\sigma_{\eta}$ becomes compressive in the middle of the membrane, while maintaining tension near the fixed boundaries. As a result, the effect of the initial stretch will become less dominant, and the magnitude of tensile $\sigma_{\eta}$ near the fixed boundaries falls off. With increasing compression in the middle, the first wrinkles are generated and the tensile $\sigma_{\eta}$ region near the fixed boundaries is broken down into multiple regions. The upward convex wrinkles will maintain the tensile region while the downward convex wrinkles will expand the compressive region to the fixed boundary.

The collapsed sections are generated only on upward convex wrinkles near the fixed boundaries. The generation starts in the compressive $\sigma_{\eta}$ region that is just above/below the tensile $\sigma_{\eta}$ region. Because of the proximity of the tensile region, compared to the downward convex wrinkles, the magnitude of compression is reduced. Additionally, the magnitude of curvature $\kappa_{\xi}$ is smaller on upward convex than on the downward convex wrinkles.

A collapsed section on a upward convex wrinkle is a downward convex deformation. The opposite applies for collapsed sections on downward convex wrinkles. By considering the magnitudes of $\sigma_{\eta}$ and $\kappa_{\xi}$, it is more difficult to form collapsed sections on downward convex wrinkles than on upward convex wrinkles.

The effects of the cylindrical shape can be summarized as follows. The curved shape of the membrane breaks the membrane symmetry with respect to the $x y$-plane. The first effect is that upward convex wrinkles have a different shape than the downward convex wrinkles. The second effect is variation in the second principal stress along the length of the fixed boundaries. The result is a state of minimum energy that is represented by collapsed sections generated at specific points on only upward convex wrinkles. The points are defined as points of minimum value of compressive $\sigma_{\eta}$ on upward convex wrinkles. Generating collapsed sections at these points in the membrane requires the least energy. 


\section{Conclusion}

In this study, the equilibrium path tracking method is used with the finite element method to obtain the wrinkling behavior of flat and cylindrical membranes undergoing shear displacement. The flat membrane is modeled using cyclic boundary conditions to generate behavior similar to the cylindrical membrane. Both membrane models undergo increasing shear displacement, and wrinkling is observed. Initially, wrinkles are formed that span the entire height of the membrane.

After these wrinkles are formed, the results for the flat membrane show a specific behavior. Wrinkle number is increased local near the fixed boundaries. This behavior is caused by a specific stress state near the fixed boundaries because the membrane out-of-plane deformation is limited. However, this stress state is constant along the fixed boundary. This allows the localized wrinkles to form freely and move in the direction of constant stress.

Similar behavior is observed near the fixed boundaries of the cylindrical membrane. Again, because the out-of-plane deformation is limited, a stress state exists that causes a local increase in wrinkle number. However, due to the cylindrical shape of the membrane, the stress state is no longer constant along the fixed boundaries. Instead, there is a variation between regions of tension and compression. This variation is fixed to the initial wrinkles. As a result, localized wrinkles cannot form freely or move along the fixed boundary. This mechanism of local wrinkle control occurs naturally on the cylindrical membrane.

\section{References}

[1] Chmielewsk,A. B., "Overview of Gossamer Structures," Gossamer Spacecraft: Membrane and Inflatable Structures Technology for Space Applications, edited by C. H. M. Jenkins, Vol. 191, Progress in Astronautics and Aeronautics, AIAA, Reston, VA, 2001, pp.1-20.

[2] Senda, K., Oda, T., Ota, S., Igarashi, Y., Watanabe, A., Hori, T., Ito, H., Tsunoda, H., and Watanabe, K., "Deploy Experiment of Inflatable Tube using Work Hardening," Proc. of AIAA Gossamer Spacecraft Forum, AIAA-2006-1808, Newport, RI, 2006, pp.1-18.

[3] Timoshenko, S.P. and Gere, J.M., "Bending of thin plates," Theory of Elastic Stability 2nd ed., Springer, New York, 2010, pp. 319-346

[4] Wong, Y.W. and Pellegrino, S., "Wrinkled Membranes, Part I," Journal of Mechanics of Materials and Structures, Vol. 1, No. 1, Jan. 2006, pp.3-25. 
[5] Wong, Y.W. and Pellegrino, S., "Wrinkled Membranes, Part II," Journal of Mechanics of Materials and Structures, Vol. 1, No. 1, Jan. 2006, pp.27-61.

[6] Wong, Y.W. and Pellegrino, S., "Wrinkled Membranes, Part III," Journal of Mechanics of Materials and Structures, Vol. 1, No. 1, Jan. 2006, pp.63-95.

[7] Cerda, E. and Mahadevan, L., "Geometry and Physics of Wrinkling," Physical Review Letters, Vol. 90, No. 7, Feb. 2003, pp.074302-1-074302-4.

doi: 10.1103/PhysRevLett.90.074302

[8] Leifer, J., Jones, D.C., Cook, A.M., "Gravity-Induced Wrinkling in Subscale, Singly Curved Parabolic Gossamer Membrane," Journal of Spacecraft and Rockets, Vol. 47, No. 1, 2010, pp. 214-219 doi: $10.2514 / 1.45672$

[9] Woo, K., Jenkins, C.H., "Effect of Crease Orientation on Wrinkle-Crease Interaction for Thin Membranes," Journal of Spacecraft and Rockets, Vol. 50, No. 5, 2013, pp. 1024-1034 doi: $10.2514 / 1 . \mathrm{A} 32183$

[10] Tessler, A., Sleight, D.W., "Geometrically Nonlinear Shell Analysis of Wrinkled Thin-Film Membranes with Stress Concentrations," Journal of Spacecraft and Rockets, Vol. 44, No. 3, 2007, pp. 582-588 doi: $10.2514 / 1.22913$

[11] Inoue, S., "Prediction Methods of Wrinkling in Thin-Membrane," Proceedings of International Symposium on Space Technology and Science, Tsukuba, Japan, 2009, pp.1-7.

[12] Senda, K., Nakanishi, K., Takagi, K., "Wrinkle Generation Mechanism without Buckling in Sheared Rectangular Membrane," Proc. of AIAA Gossamer Spacecraft Forum, AIAA-2012-1919, Honolulu, HI, 2012, pp.1-30.

[13] Senda, K., Petrovic, M., Nakanishi, K., "Behavior of a Sheared Square Membrane with Cyclic Boundary Conditions during Wrinkling," Proc. of AIAA Gossamer Spacecraft Forum, AIAA-2013-1927, Boston, MA, 2013, pp.1-33.

[14] Endo, A., Hangai, Y., and Kawamata, S., "Post-Buckling Analysis of Elastic Shells of Revolution by the Finite Element Method," Report of Institute of Industrial Science, UDC 624.074.4:624.075.4, University of Tokyo, December 1976

[15] Wriggers, P., Wanger, W., and Miehe, C., "A Quadratically Convergent Procedure for the Calculation of Stability Points in Finite Element Analysis," Computer Methods in Applied Mechanics and Engineering, Vol. 70, No. 3, 1988, pp.329-347. doi: $10.1016 / 0045-7825(88) 90024-2$

[16] Senda, K., Petrovic, M., Nakanishi, K., "Wrinkle Generation without Bifurcation in a Shear-Enforced 
Rectangular Membrane with Free Boundaries," Journal of Spacecraft and Rockets, accessed February $27,2015$.

doi: http://arc.aiaa.org/doi/abs/10.2514/1.A33018

[17] Hoyle, R., "A bit of group theory," Pattern Formation Cambridge University Press, New York, 2007, pp. $52-84$

[18] Reddy, B.S., Chatterji, B. N., "An FFT-based technique for translation, rotation, and scale-invariant image registration," IEEE Transactions on Image Processing 5, Vol. 5, No. 8, 1996, pp.1266-1271 doi: $10.1109 / 83.506761$ 


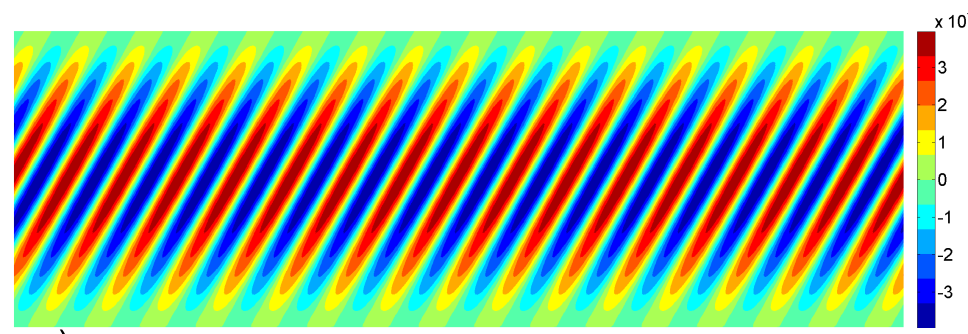

a)

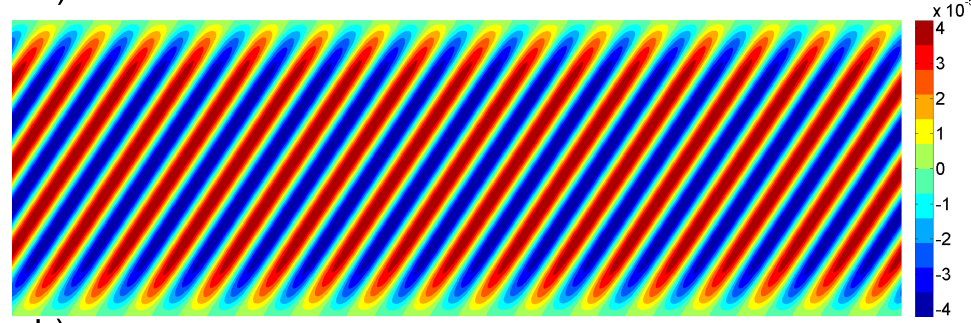

b)
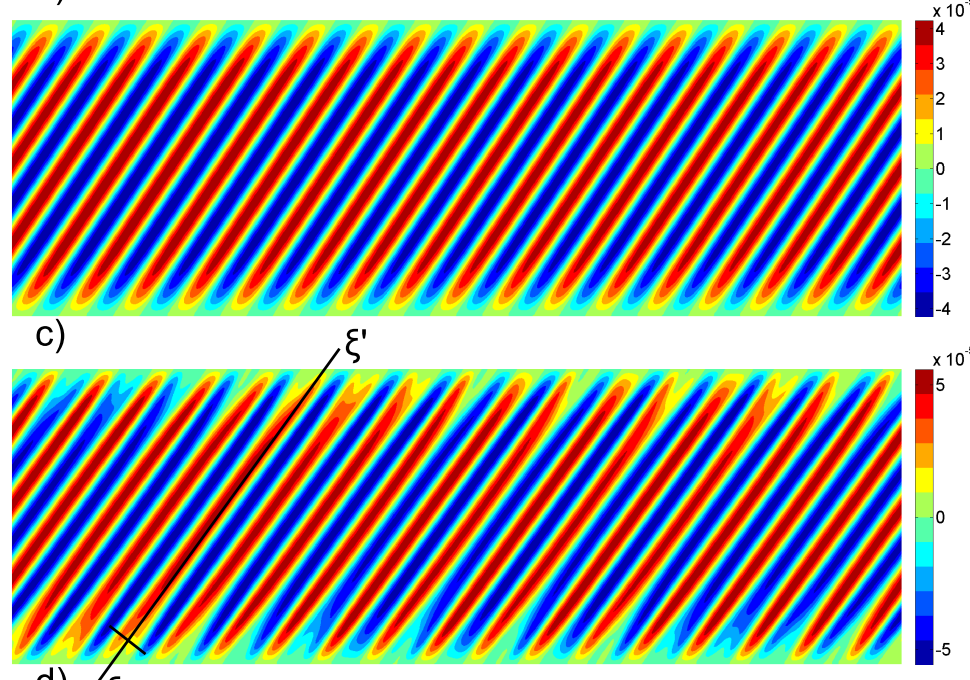

d) $\xi$

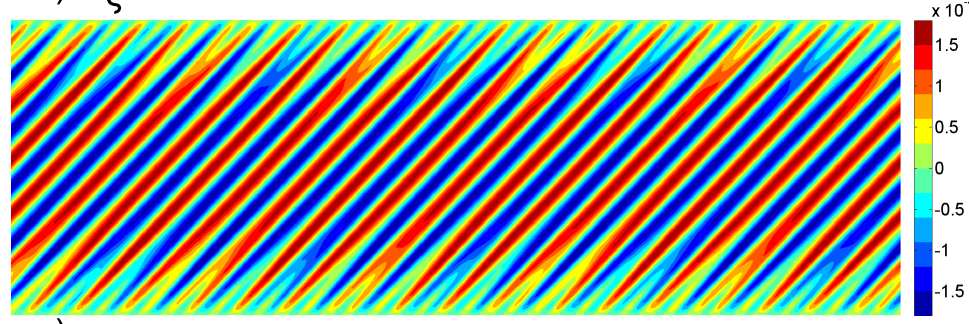

e)

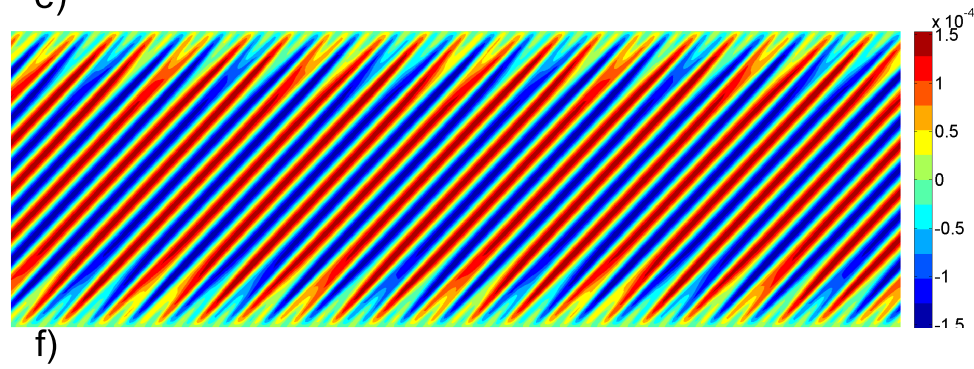

Fig. 6 Out-of-plane deformation; a) Bif. 48, b) Bif. 67, c) Bif. 69, d) Path 69-0 at $\delta u_{x}=$ $85.00 \times 10^{-6}[\mathrm{~m}]$, e) Path 69-0 at $\delta u_{x}=500.00 \times 10^{-6}[\mathrm{~m}]$, f) Bif. 500. 


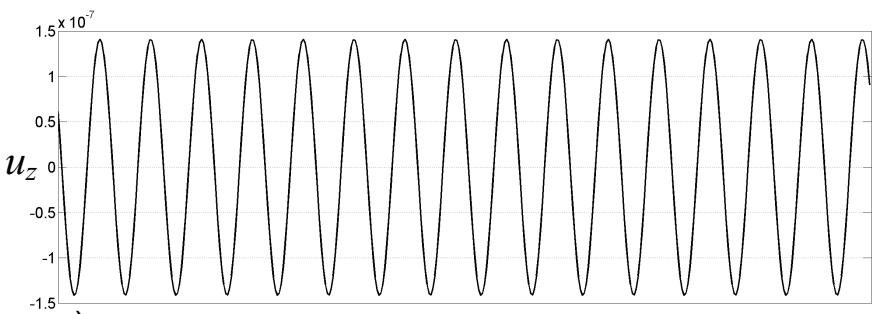

a)

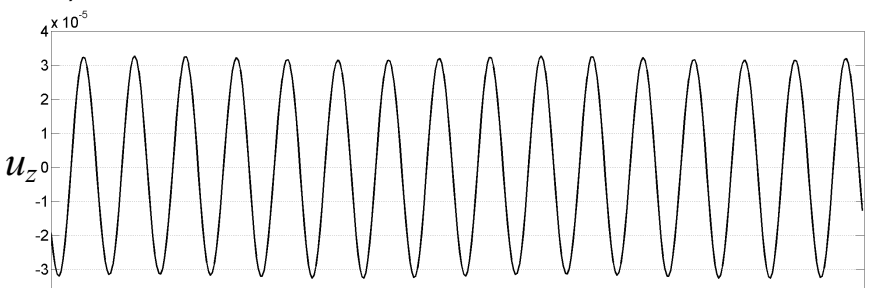

b)

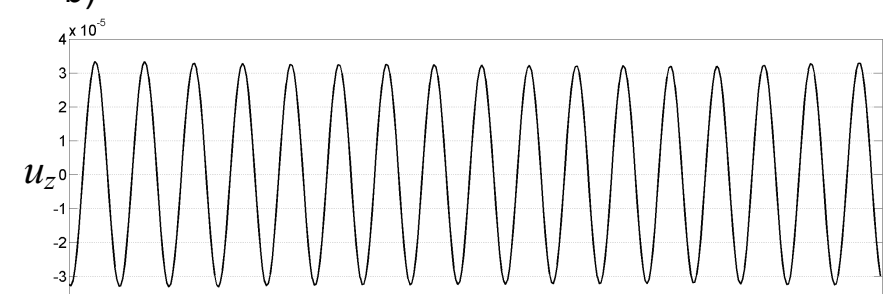

C)

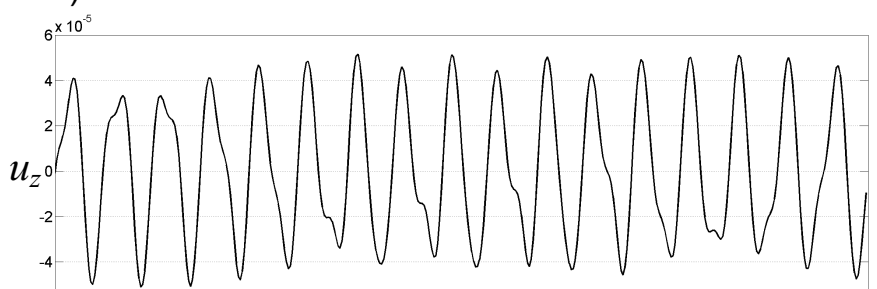

d)

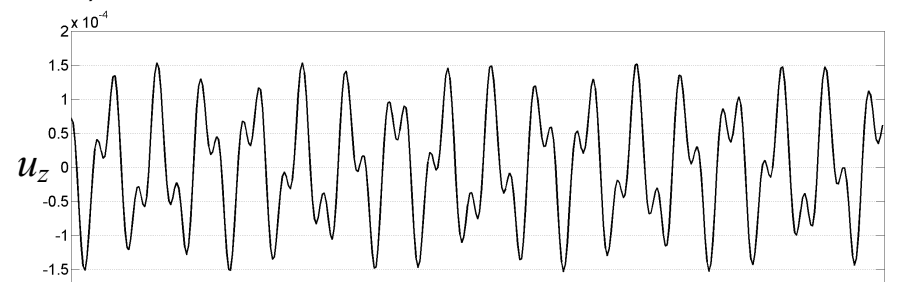

$\left.{ }^{-2} e\right)$

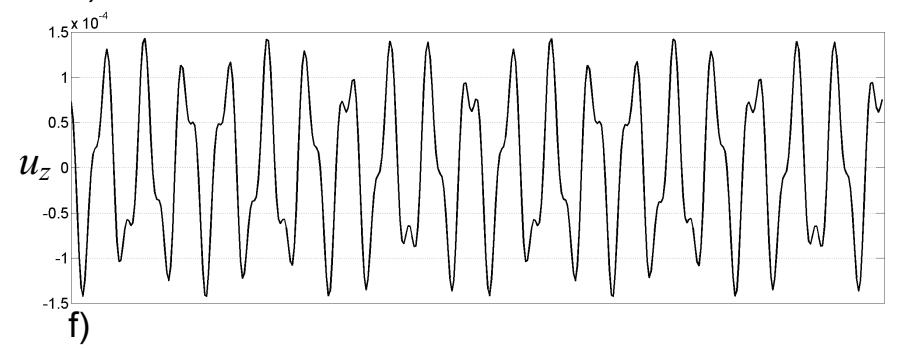

Fig. 7 Out-of-plane deformation in section B-B'; a) Bif. 48, b) Bif. 67, c) Bif. 69, d) Path 69-0 at $\delta u_{x}=85.00 \times 10^{-6}[\mathbf{m}]$, e) Path 69-0 at $\delta u_{x}=500.00 \times 10^{-6}[\mathbf{m}]$, f) Bif. 500 . 


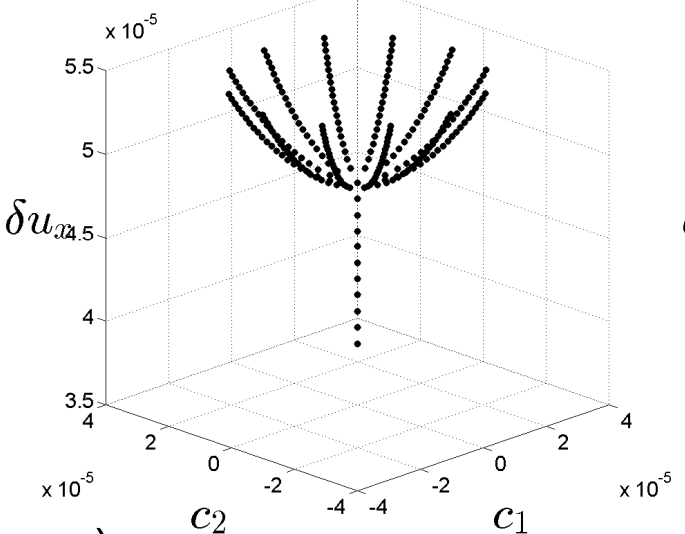

a)
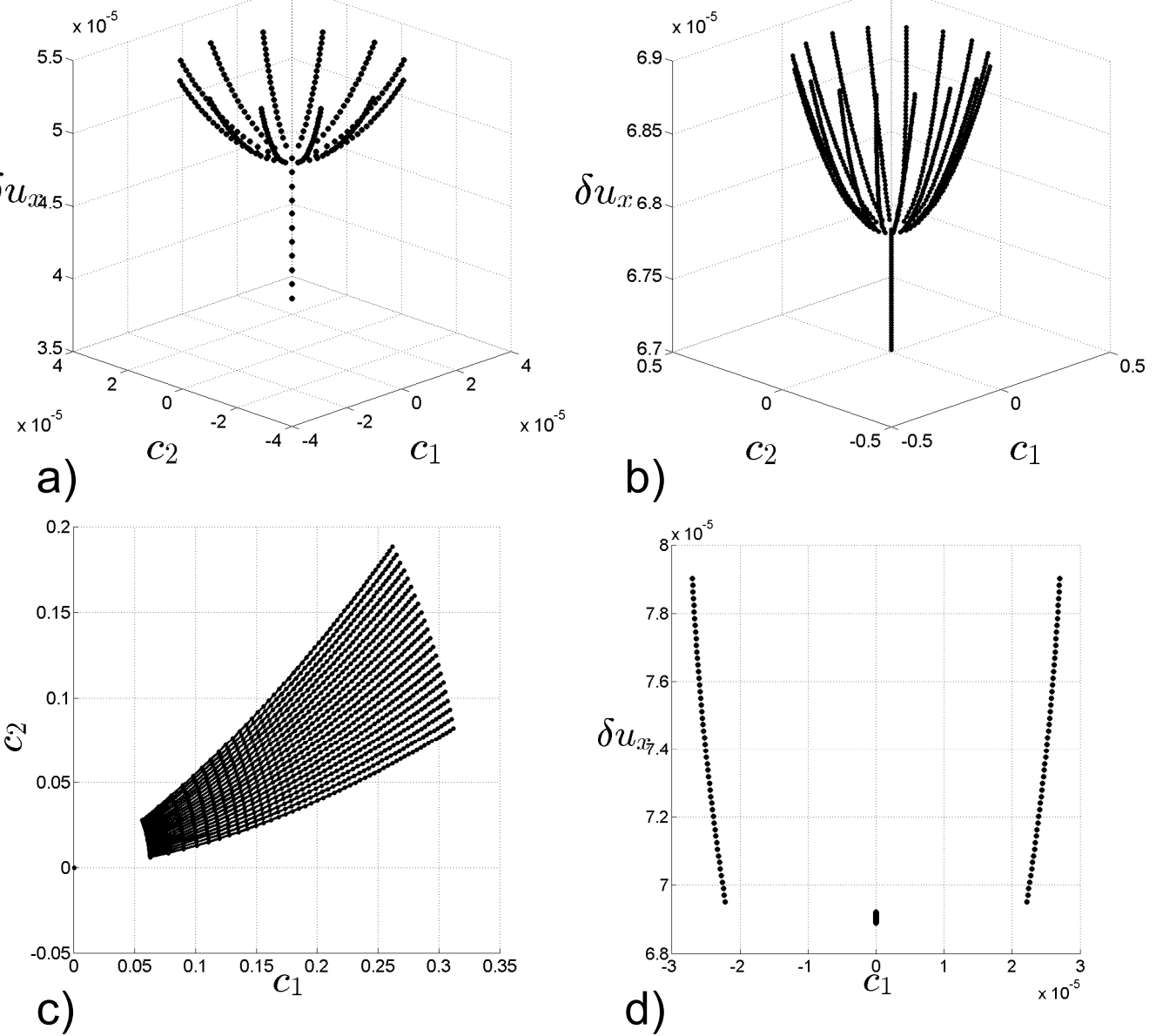

b)

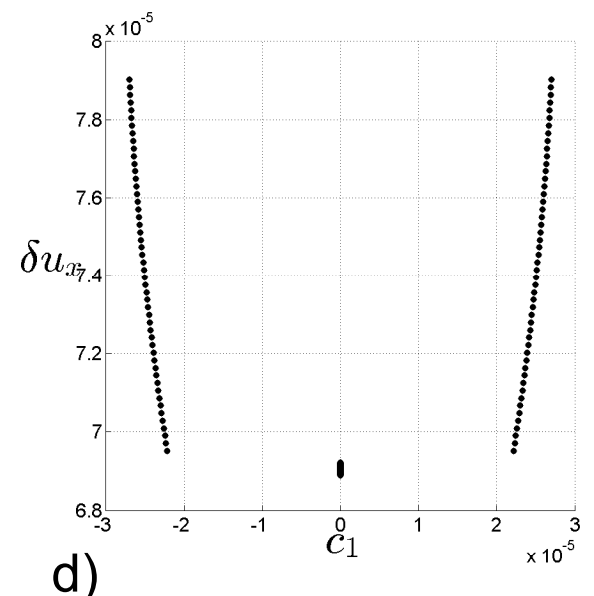

Fig. 8 Bifurcation diagrams for the flat membrane; a) Bif. 48, b) Bif. 67, c) close up after Bif. 67, d) Bif. 69. 

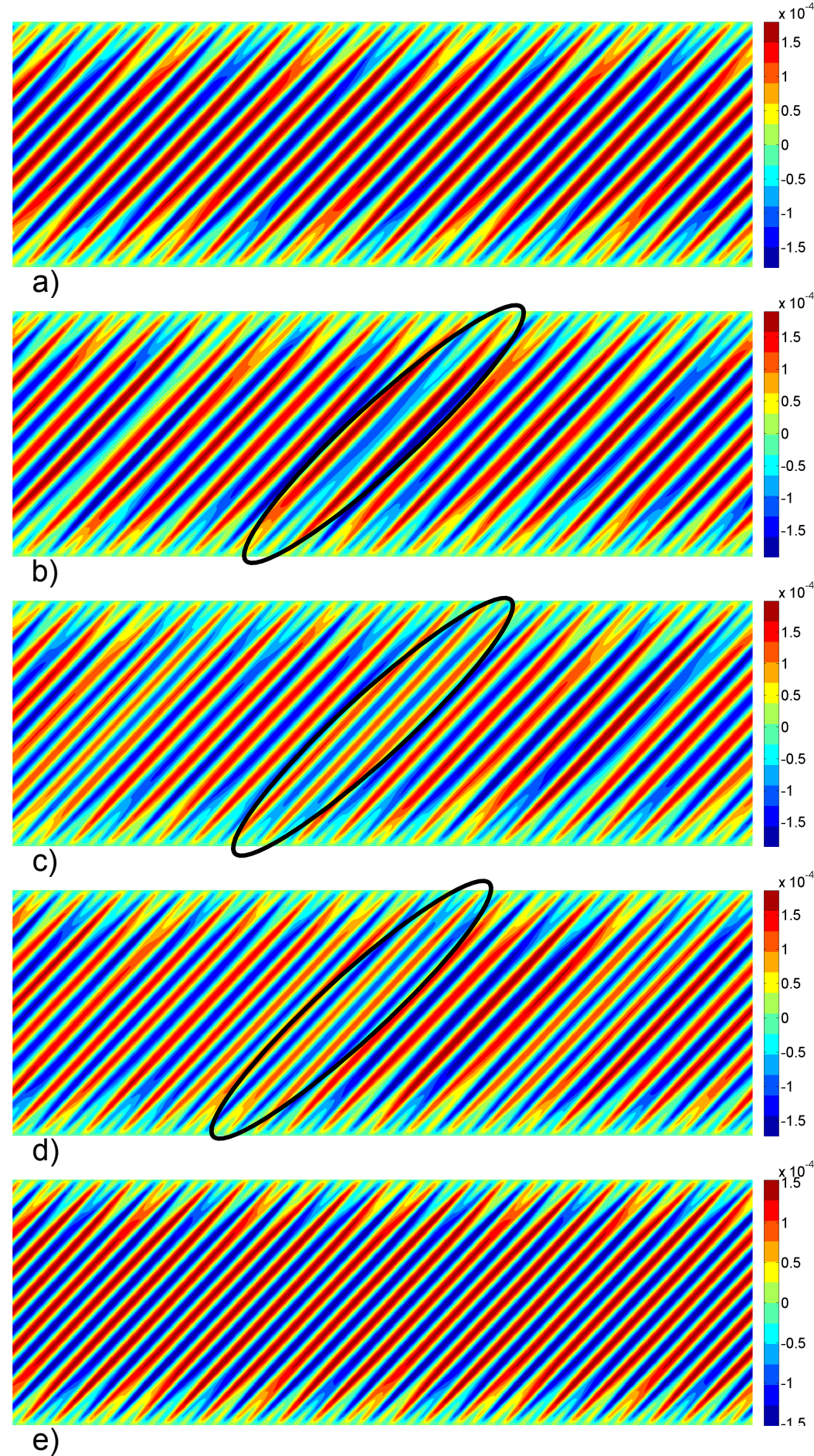

Fig. 9 Wrinkle split, Bif. 500, flat membrane: a) initial condition, b) start of collapsed section expansion, c) wrinkle splitting, d) new wrinkle growth, e) final pattern 

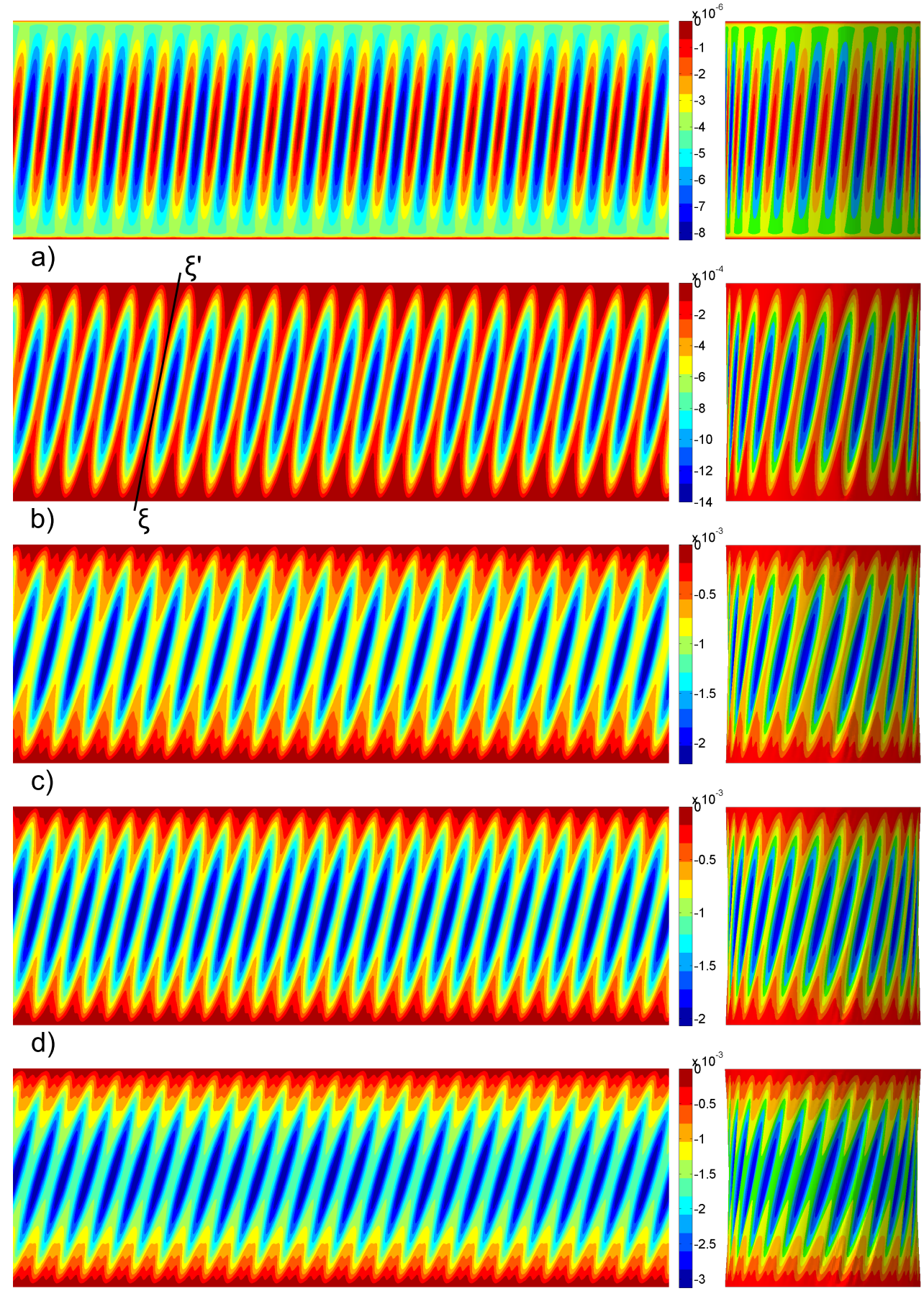

d)

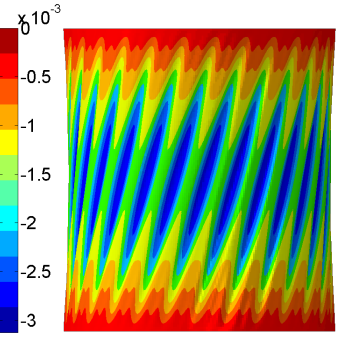

e)

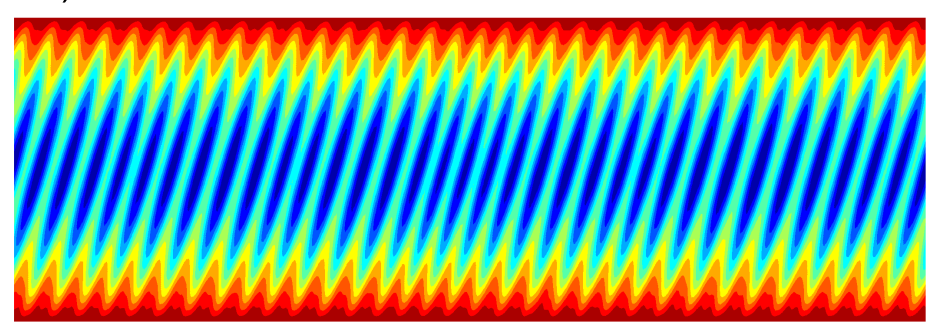

f)

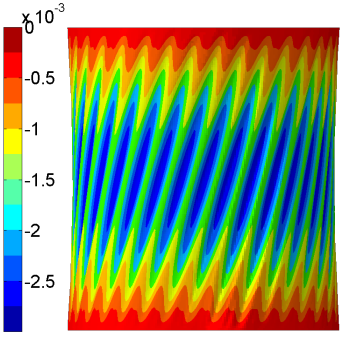

Fig. 10 Out-of-plane deformation; a) Bif. 11, b) Path 11-0 at $\delta u_{x}=432.00 \times 10^{-6}[\mathbf{m}]$, c) Path 11-0 at $\delta u_{x}=1068.00 \times 10^{-6}[\mathbf{m}]$, d) Bif. 1068, e) Path 1068-0 at $\delta u_{x}=2178.00 \times 10^{-6}[\mathbf{m}]$, f) Bif. 2178. 

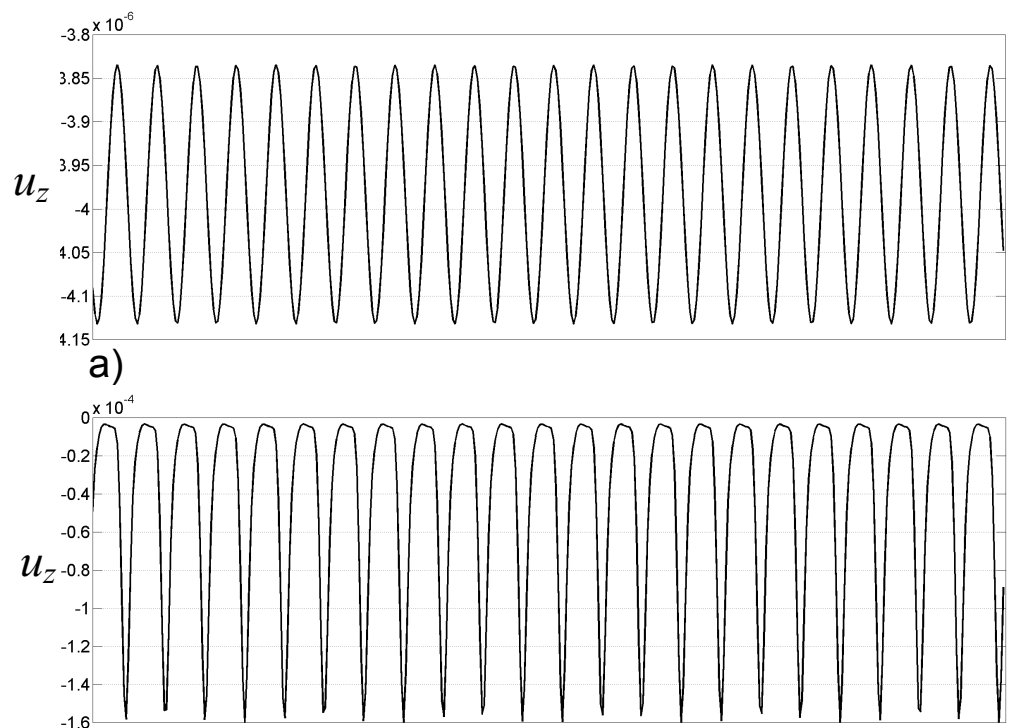

b)
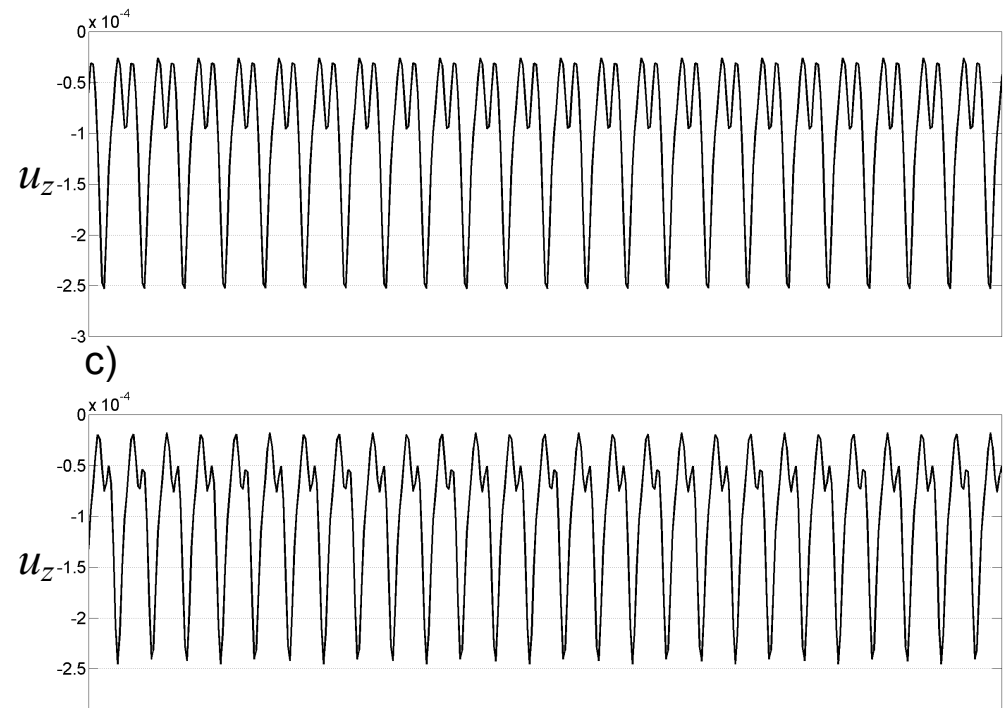

d)

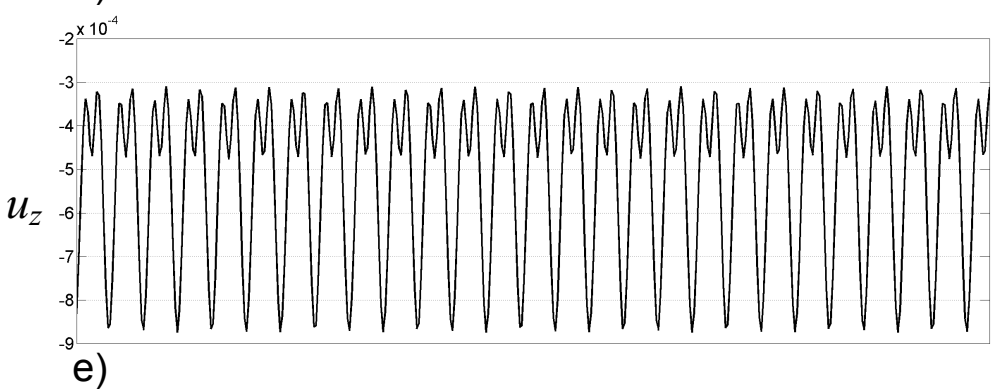

Fig. 11 Out-of-plane deformation in section BB'; a) Bif. 11, b) Path 11-0 at $\delta u_{x}=432.00 \times$ $10^{-6}[\mathrm{~m}]$, c) Path 11-0 at $\delta u_{x}=1068.00 \times 10^{-6}[\mathbf{m}]$, d) Bif. 1068, e) Path 1068-0 at $\delta u_{x}=2178.00 \times$ $10^{-6}[\mathbf{m}]$. 


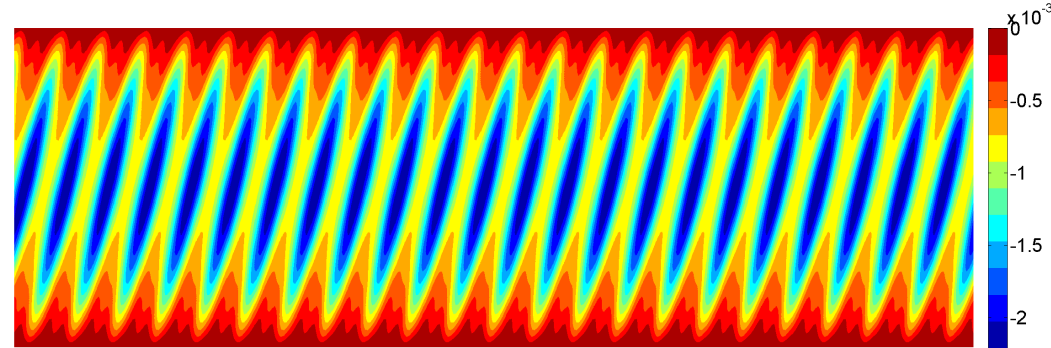

a)

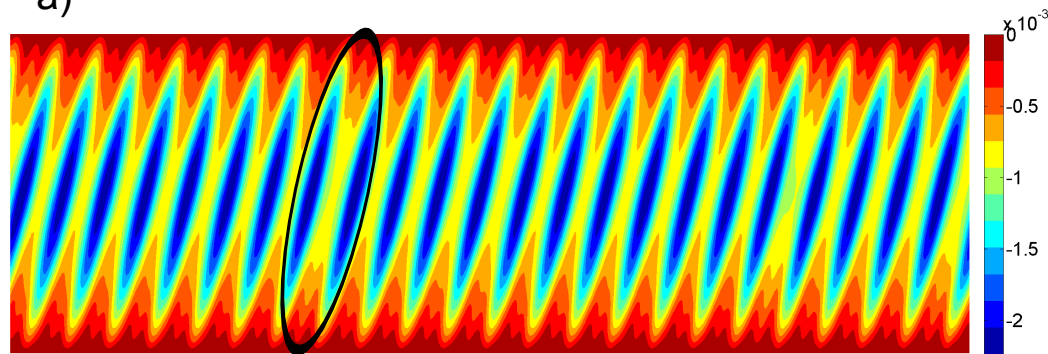

b)

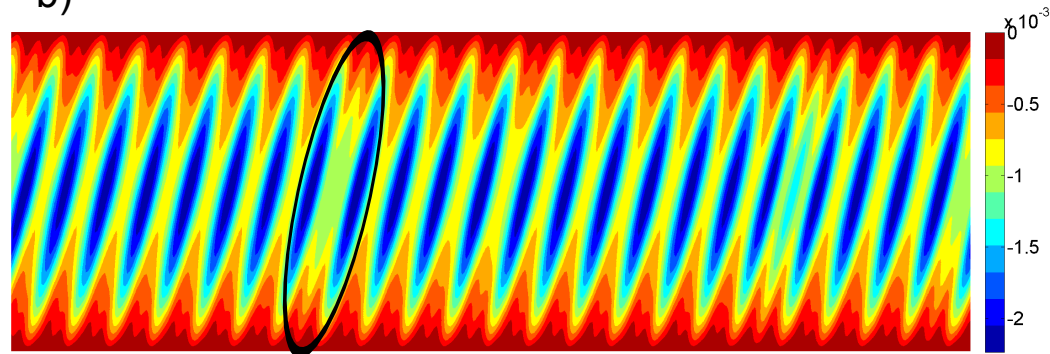

c)

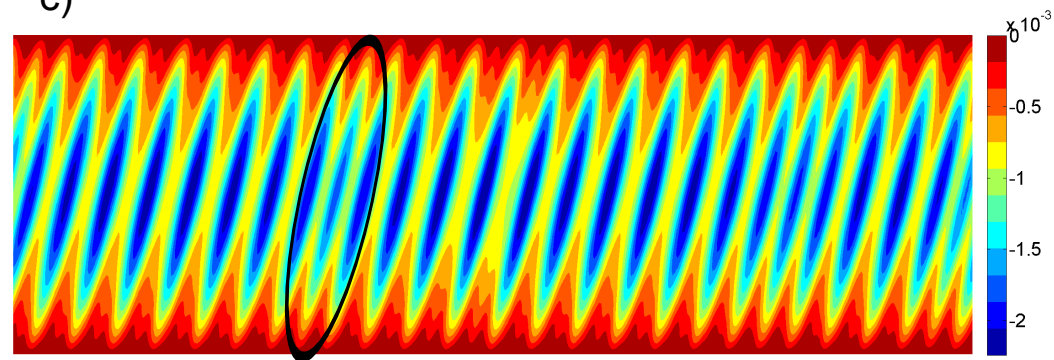

d)

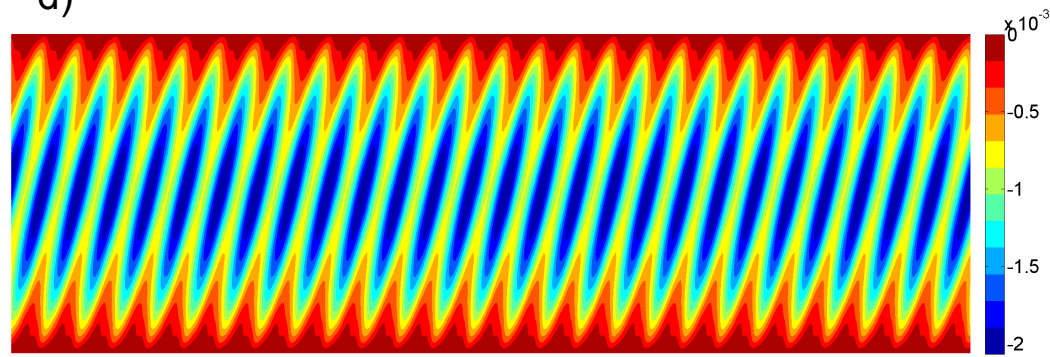

e)

Fig. 12 Wrinkle split, Bif. 1068; a) initial wrinkle pattern, b) start of collapsed section expansion, c) formation of a new downward convex wrinkle, d) growth of a downward convex wrinkle, e) final wrinkle pattern. 


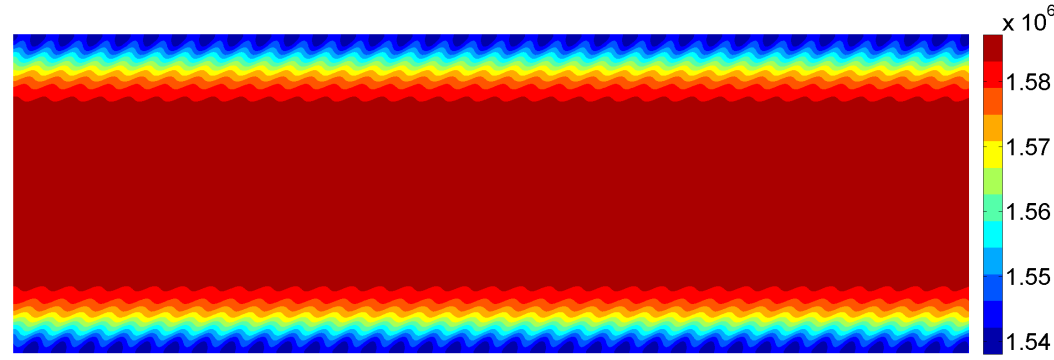

a)

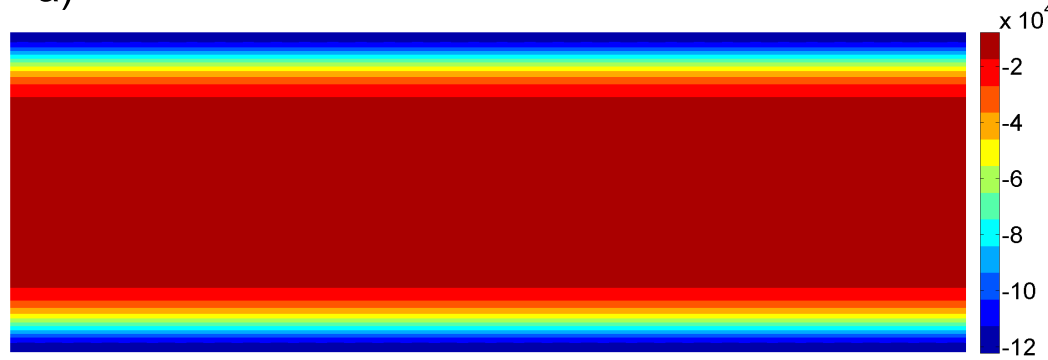

b)

Fig. 13 Flat membrane principal stress; a) $\sigma_{\xi}$, b) $\sigma_{\eta}$.

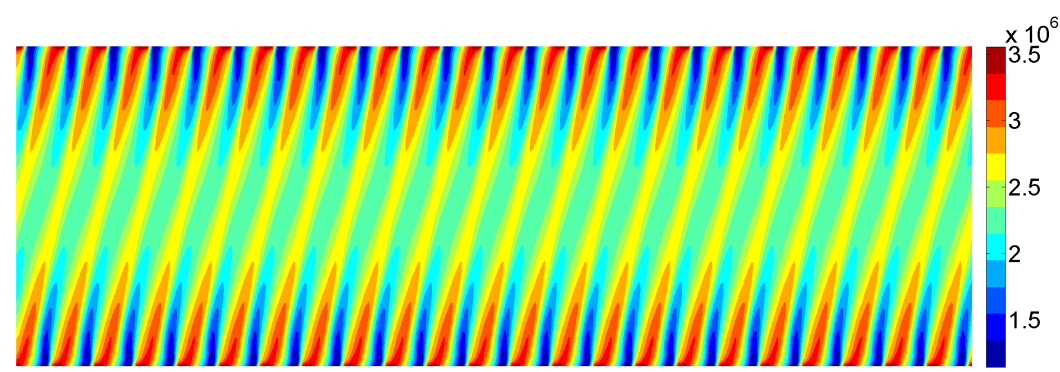

a)

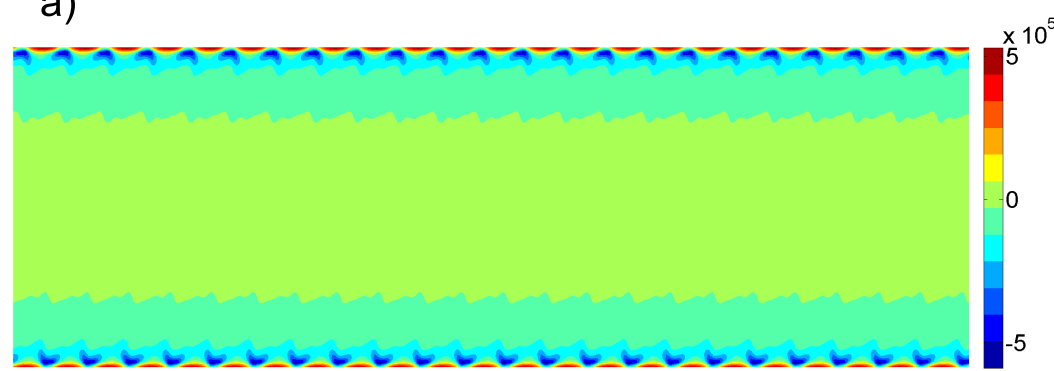

b)

Fig. 14 Cylindrical membrane principal stress; a) $\sigma_{\xi}$, b) $\sigma_{\eta}$. 

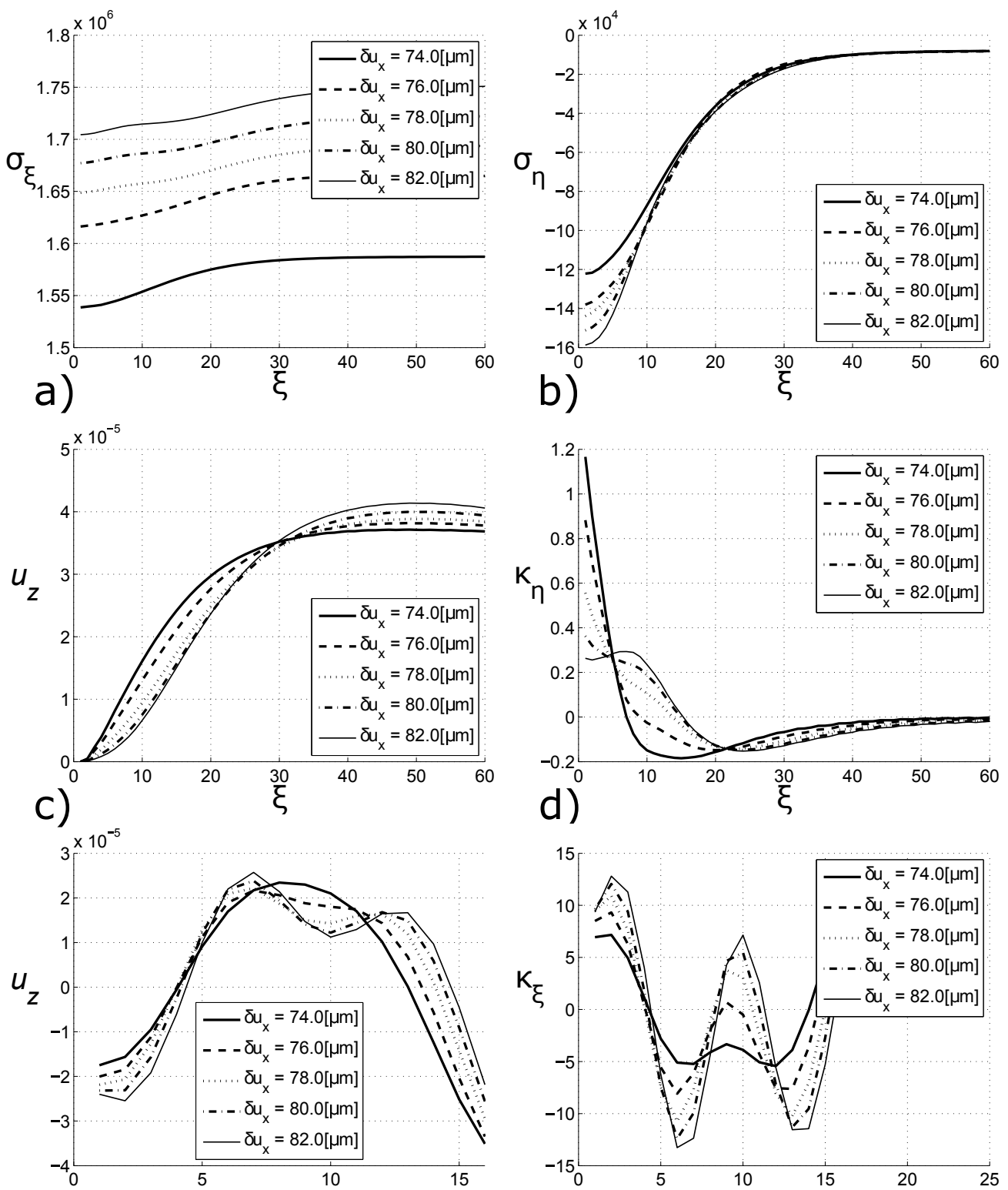

e)

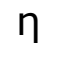

f)
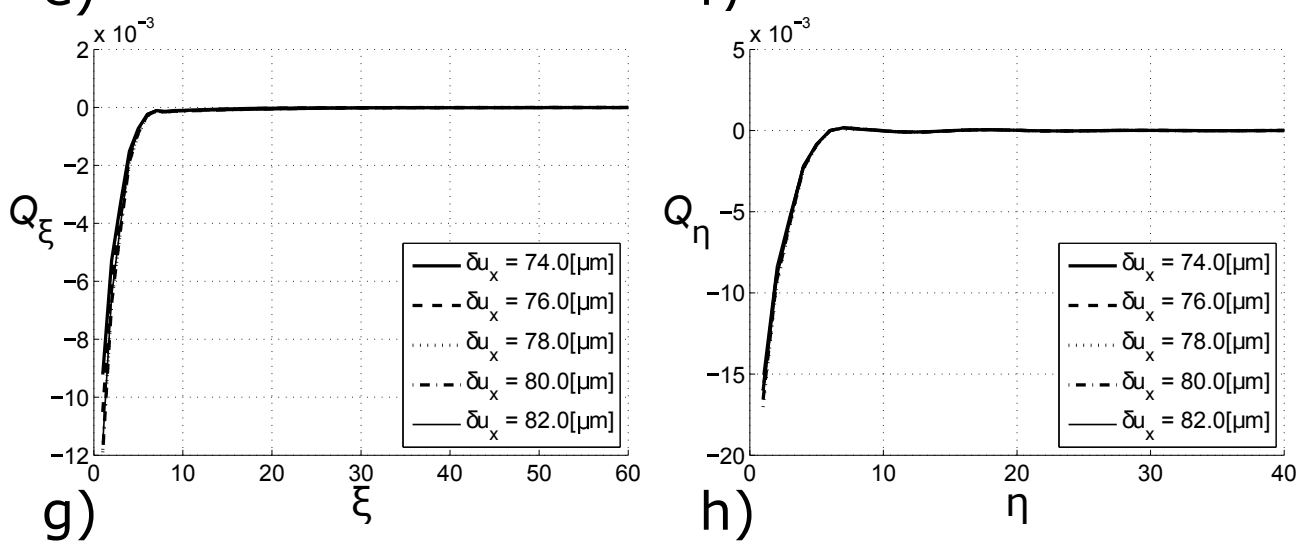

Fig. 15 Flat membrane collapsed section; a) $\sigma_{\xi}$ at section $\xi \xi^{\prime}$, b) $\sigma_{\eta}$ at section $\xi \xi^{\prime}$, c) $u_{z}$ at section $\xi \xi^{\prime}$, d) $\kappa_{\eta}$ at section $\xi \xi^{\prime}$, e) $u_{z}$ at section $\eta \eta^{\prime}$, f) $\kappa_{\xi}$ at section $\eta \eta^{\prime}$, g) $Q_{\xi}$ at section $\left.\xi \xi^{\prime}, \mathbf{h}\right)$ at section $\eta \eta^{\prime}$. 

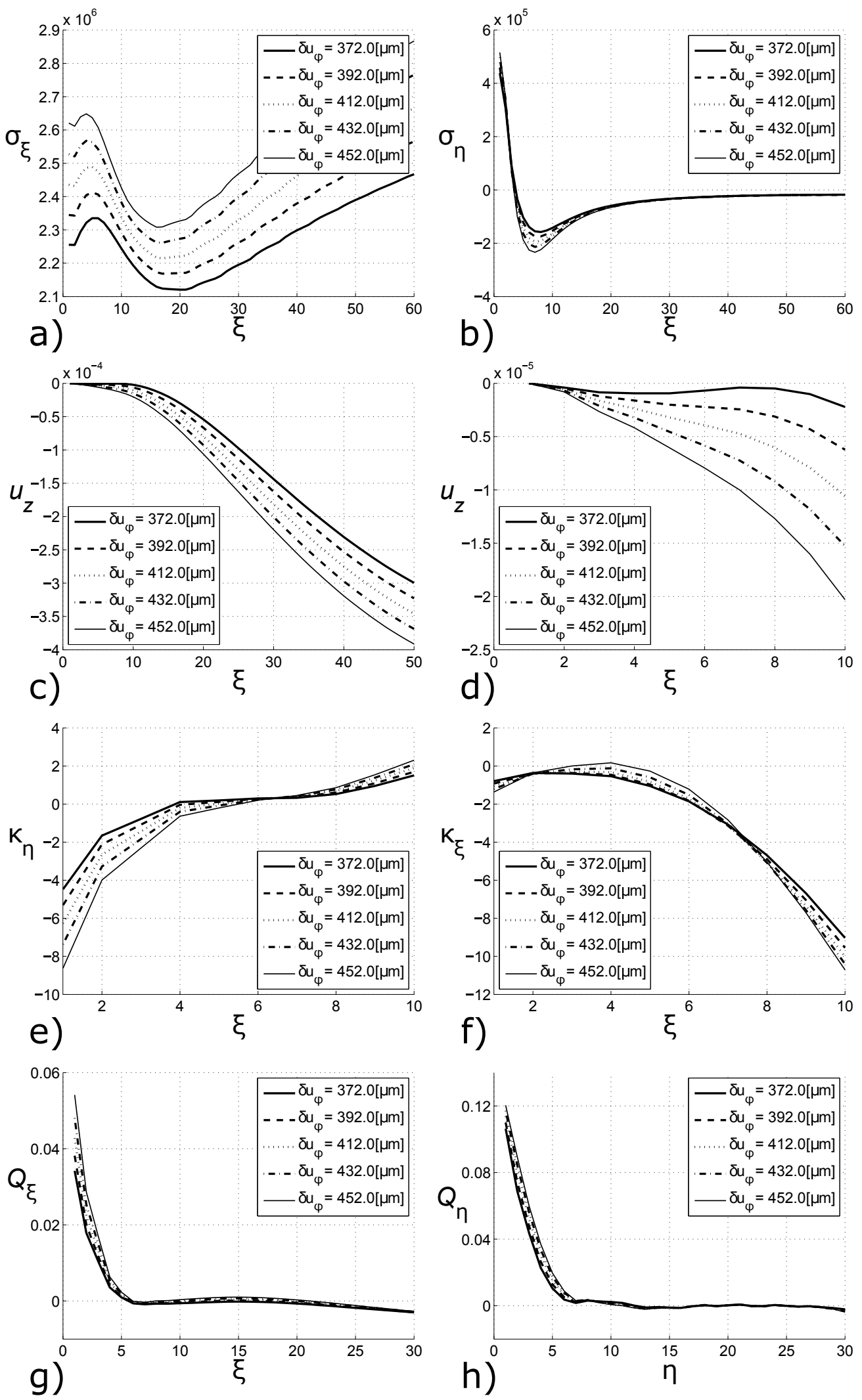

Fig. 16 Cylindrical membrane collapsed section; a) $\sigma_{\xi}$ at section $\xi \xi^{\prime}$, b) $\sigma_{\eta}$ at section $\xi \xi^{\prime}$, c) $u_{z}$ at section $\xi \xi^{\prime}$, d) $u_{z}$ at section $\xi \xi^{\prime}$, e) $\kappa_{\eta}$ at section $\xi \xi^{\prime}$, f) $\kappa_{\xi}$ at section $\xi \xi^{\prime}$, g) $Q_{\xi}$ at section $\xi \xi^{\prime}$, h) $Q_{\eta}$ at section $\eta \eta^{\prime}$. 


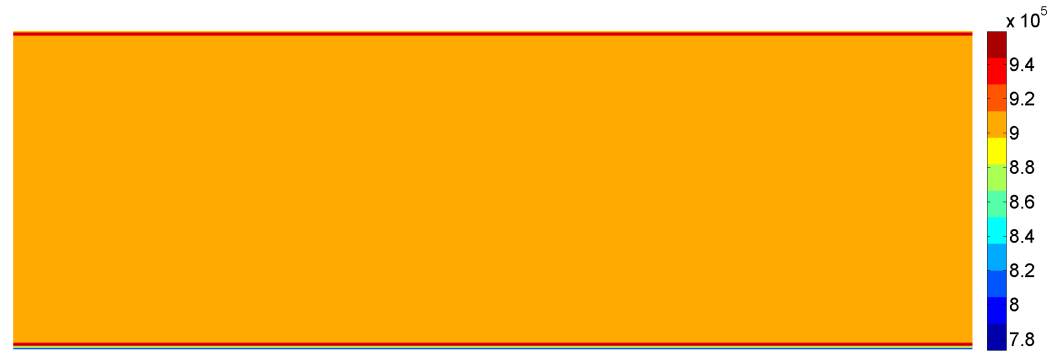

a)

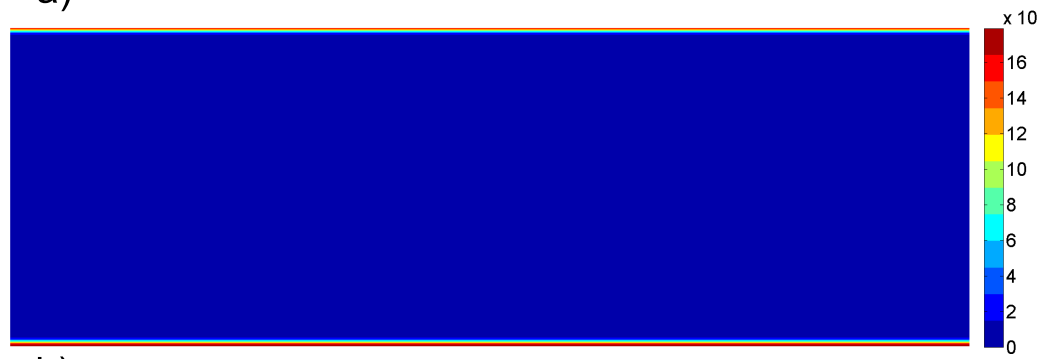

b)

Fig. 17 Cylindrical membrane principal stress before shear; a) $\sigma_{\xi}$, b) $\sigma_{\eta}$. 